OPEN ACCESS

Edited by:

Ashok Kumar,

University of Florida, United States

Reviewed by:

Rogier Landman,

Broad Institute, United States

Stephen Aita,

Dartmouth College, United States

${ }^{*}$ Correspondence:

Matteo De Marco

matteo.demarco@brunel.ac.uk

Received: 10 March 2021

Accepted: 09 July 2021

Published: 03 August 2021

Citation:

De Marco M. Blackburn DJ and Venneri A (2021) Serial Recall Order and Semantic Features of Category Fluency Words to Study Semantic Memory in Normal Ageing. Front. Aging Neurosci. 13:678588. doi: 10.3389/fnagi.2021.678588

\section{Serial Recall Order and Semantic Features of Category Fluency Words to Study Semantic Memory in Normal Ageing}

\author{
Matteo De Marco ${ }^{1,2 *}$, Daniel J. Blackburn² and Annalena Venneri1,2 \\ ${ }^{1}$ Department of Life Sciences, Brunel University London, London, United Kingdom, ${ }^{2}$ Department of Neuroscience, The \\ University of Sheffield, Sheffield, United Kingdom
}

Background: Category Fluency Test (CFT) is a common measure of semantic memory (SM). Test performance, however, is also influenced by other cognitive functions. We here propose a scoring procedure that quantifies the correlation between the serial recall order (SRO) of words retrieved during the CFT and a number of linguistic features, to obtain purer SM measures. To put this methodology to the test, we addressed a proofof-concept hypothesis whereby, in alignment with the literature, older adults would show better SM.

Methods: Ninety participants (45 aged 18-21 years; 45 aged 70-81 years) with normal neurological and cognitive functioning completed a 1-min CFT. SRO was scored as an ordinal variable incrementing by one unit for each valid entry. Each word was also scored for 16 additional linguistic features. Participant-specific normalised correlation coefficients were calculated between SRO and each feature and were analysed with group comparisons and graph theory.

Results: Younger adults showed more negative correlations between SRO and "valence" (a feature of words pleasantness). This was driven by the first five words generated. When analysed with graph theory, SRO had significantly higher degree and lower betweenness centrality among older adults.

Conclusion: In older adults, SM relies significantly less on pleasantness of entries typically retrieved without semantic control. Moreover, graph-theory metrics indicated better optimised links between SRO and linguistic features in this group. These findings are aligned with the principle whereby SM processes tend to solidify with ageing. Although additional work is needed in support of an SRO-based item-level scoring procedure of CFT performance, these initial findings suggest that this methodology could be of help in characterising SM in a purer form.

Keywords: semantic memory, efficiency, centrality, hippocampus, Alzheimer's disease, pre-clinical

Abbreviations: CFT, category fluency test; SRO, serial recall order. 


\section{INTRODUCTION}

Beyond its use in linguistics and neurology as a term to indicate the flow of language, verbal fluency identifies a cognitive ability that supports retrieval from memory (Patterson, 2011) and that is commonly used to assess semantic memory (SM). Measures of SM are particularly important to the study of cognitive ageing. Findings from large cohorts of asymptomatic adults followed up longitudinally, in fact, have revealed that performance on a major SM test, the "Category Fluency Test" (CFT) (inclusive of its analogues, e.g., the "Isaacs Set Test"), is among the earliest predictors of future progression to Alzheimer's disease (Amieva et al., 2008; Payton et al., 2020). Conversely, a large body of evidence indicates that SM tends to be largely preserved and even improve with healthy ageing (Nyberg et al., 1996, 2003; Park et al., 2002; Verhaeghen, 2003; Rönnlund et al., 2005; Small et al., 2011). Although a decrease in performance has been frequently reported in older adults on the CFT, this is thought, however, to be accounted for by decline of other supportive abilities such as executive functioning and processing speed (Spaan, 2015; Aita et al., 2019; Gonzalez-Burgos et al., 2019). In this respect, although CFT performance is widely regarded, for all intents and purposes, as an index of SM (Venneri et al., 2016, 2018), a number of studies have included it as part of the assessment of executive functioning (Rende et al., 2002; Gibbons et al., 2012). Executive abilities, in fact, go further than providing simple external facilitatory resources to task engagement. SM, in fact, relies on an intrinsic executive component, "semantic control", that supports manipulation of semantic content to facilitate retrieval (Lambon Ralph et al., 2017). In addition, performance on this test is also influenced by other functions such as processing speed (Elgamal et al., 2011) and episodic memory (Greenberg et al., 2009). Furthermore, clinicians often consider CFT scores as reflecting expressive language abilities, since disrupted SM retrieval affects linguistic production and may interfere with effective communication. Although this evidence clearly indicates that the CFT has been thoroughly investigated in relation to a variety of cognitive functions, no conclusive framework has yet been outlined and no study has quantified the contribution of each distinct function to test performance in the context of ageing.

There is a clinical interest in assessing SM in the most accurate possible way. The latest clinical diagnostic guidelines for Alzheimer's disease discourage the use of available biomarkers as the sole diagnostic features at the pre-clinical stage (Dubois et al., 2021). It is thus of central importance to explore alternative methodological routes that can help identify subtle changes indicative of early stage neurodegeneration. In this respect, SM may play a crucial role (Venneri et al., 2016). Alternative methodologies have been studied to overcome the multi-componential element that characterises the construct validity of standard CFT scoring, to obtain "purer" measures of SM. A large number of studies have investigated the semantic properties of words generated during performance on CFTs, such as "age of acquisition," "typicality," and "frequency," i.e., "item-level features" (Forbes-McKay et al., 2005; Biundo et al., 2011; Venneri et al., 2011; Vita et al., 2014; Quaranta et al.,
2016; Wakefield et al., 2018; Vonk et al., 2019a,b; Taler et al., 2020), under the assumption that the ability to generate less frequent, less typical and later acquired words would reflect efficient semantic processing (Murray and Forster, 2004; Steyvers and Tenenbaum, 2005; Plant et al., 2011). Other studies have focussed on the semantic relationships between words (e.g., Goñi et al., 2011; Pakhomov et al., 2012; Bertola et al., 2014; Quaranta et al., 2019), on the assumption that the sequence of words could be indicative of the integrity of the underlying semanticprocessing system.

In this exploratory study we combined the principles of itemlevel and sequence-related properties to test a novel approach to CFT scoring that combines aspects of semantic processing with a property of memory retrieval. Specifically, we focussed on the positional order with which words are retrieved from memory during the process of word generation required by the test (i.e., first word recalled, second word recalled, third word recalled...), the serial recall order (SRO). The SRO score (Figure 1A) is operationalisable as an ordinal variable ranging from 1 (first word generated) and incrementing by one unit up to $n$ (nth word generated). Typically, words with higher frequency of use in a given language are generated during the first temporal segment of the minute trial (Crowe, 1998), suggesting a negative association between SRO and frequency (i.e., as the positional order increases, less frequent words are generated). This indicates that, as the category is explored in greater depth as part of the test, words generated toward the end of the trial tend to become "more difficult" exemplars, at least as far as frequency is concerned (i.e., Figures 1B,C). Moreover, a recent study found that, as categories are explored, more original entries tend to be generated, i.e., words given by less than $5 \%$ of the target cohort (Murphy and Castel, 2020).

To capture the association between SRO (a property of memory retrieval) and word features such as frequency, typicality or age of acquisition (properties of semantic processing), we calculated a series of subject-specific coefficients of correlation that quantify the trend shown by a participant's word production becoming "more difficult" as more entries are generated. We assumed that the idea of "getting more difficult" would translate into decreasing word frequency, decreasing typicality, increasing age of acquisition and further increases or decreases in a number of semantic properties (described in section "CFT-Scoring Procedures") linked to the target category (e.g., "animals"). We propose that these correlations capture the interplay of memory retrieval and semantic processing, and that aspects of SM are expressed by this interplay (i.e., as illustrated schematically in Figure 1). Supporting functions such as processing speed or executive functioning are well known to have a significant impact on word count (Rende et al., 2002; Elgamal et al., 2011; Gibbons et al., 2012). As long as correlations are stable (i.e., based on a sufficiently large sample size), however, they can be equally calculated regardless of the exact number of entries. Based on this, we formulated a first, methodological hypothesis: supporting functions will show a statistical effect on the number of valid words generated via semantic control and via control of retrieval processes, but not on the interplay between SRO and semantic features. 


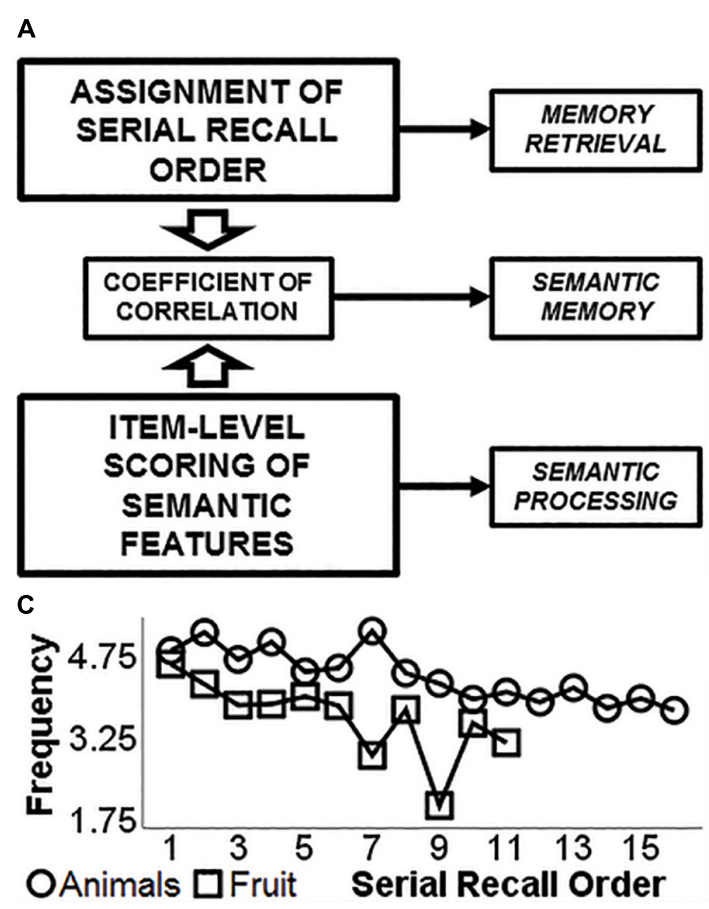

B

\begin{tabular}{|c|c|c|}
\hline $\begin{array}{l}\text { STANDARDISED } \\
\text { ENTRY }\end{array}$ & $\begin{array}{c}\text { SERIAL } \\
\text { RECALL ORDER }\end{array}$ & $\begin{array}{l}\text { FEATURE SCORE } \\
\text { (e.g., frequency) }\end{array}$ \\
\hline CAT & 1 & 4.830 \\
\hline DOG & 2 & 5.168 \\
\hline SHEEP & 3 & 4.667 \\
\hline HORSE & 4 & 4.990 \\
\hline cow & 5 & 4.440 \\
\hline PIG & 6 & 4.511 \\
\hline FISH & 7 & 5.192 \\
\hline MOUSE & 8 & 4.415 \\
\hline RAT & 9 & 4.226 \\
\hline SQUIRREL & 10 & 3.936 \\
\hline OWL & 11 & 4.070 \\
\hline PENGUIN & 12 & 3.881 \\
\hline WHALE & 13 & 4.145 \\
\hline DOLPHIN & 14 & 3.770 \\
\hline CROCODILE & 15 & 3.948 \\
\hline PANDA & 16 & 3.730 \\
\hline APPLE & 1 & 4.584 \\
\hline BANANA & 2 & 4.215 \\
\hline PEAR & 3 & 3.826 \\
\hline PINEAPPLE & 4 & 3.845 \\
\hline STRAWBERRY & 5 & 3.986 \\
\hline RASPBERRY & 6 & 3.814 \\
\hline PAPAYA & 7 & 2.900 \\
\hline MANGO & 8 & 3.743 \\
\hline PASSIONFRUIT & 9 & 1.974 \\
\hline MELON & 10 & 3.492 \\
\hline WATERMELON & 11 & 3.132 \\
\hline
\end{tabular}

Spearman's $r$ o $=-0.539, p=0.004$

FIGURE 1 | Graphical representation of the principle at the basis of the study. While the serial order of recall is a property of memory retrieval, features such as word "frequency," "typicality," or "age of acquisition" are linked to semantic processing. The calculation of a coefficient of correlation between these two variables would produce an index that can inform how retrieval from memory is associated with semantic "difficulty" of words, and thus provide a theoretically valid measure of semantic memory (A). On the right, a practical example of feature-to-feature correlation between "serial recall order" and "frequency" (B). This is illustrated in the bottom left corner (C).

We then relied on this framework to test a second, experimental hypothesis designed ad hoc and meant to lay the thematic foundations for this line of research. To this end, we analysed retrospectively the CFT performance of 45 younger adults and 45 older adults. Since, as highlighted by the literature, SM tends to consolidate with ageing (Nyberg et al., 1996, 2003; Park et al., 2002; Verhaeghen, 2003; Rönnlund et al., 2005; Small et al., 2011), we expected that this set of correlation coefficients would show significant group differences indicating higher levels of semantic organisational structure among older adults. Older adults would thus show significantly stronger correlations in the same direction (i.e., positive or negative) as that shown by younger adults (e.g., among others, a significantly stronger negative correlation between SRO and typicality and between SRO and frequency, and a significantly stronger positive correlation between SRO and age of acquisition would be expected). To address this hypothesis, we tested for group differences via the direct comparison of standardised coefficients of correlation and via the exploratory analysis of nodal properties of SRO, as informed by graph theory.

\section{MATERIALS AND METHODS}

\section{Participants}

This study is based on the secondary analysis of datasets collected on cognitively normal volunteers. These had been originally recruited as part of a large cohort for the purpose of collecting in-house normative data for neuropsychological test scores, to be used as numerical reference to aid profiling of neurological patients in tertiary care. Two distinct age groups were targeted in this study (Table 1): volunteers between 18 and 21 years of age (henceforth, "younger adults") and between 70 and 81 (henceforth, "older adults"). The choice of comparing two distant age groups was guided by normative studies of CFT [see (Woods et al., 2016) for a study carried out in English native speakers]: these studies show that CFT performance across the entire adulthood can be accounted for by a single normative model.

A screening questionnaire was completed by each participant prior to recruitment to rule out exclusion criteria of medical or psychological nature that might otherwise have had an impact on neurological and cognitive profiles. These included: diagnostic entities or clinical signs mechanistically linked to psychological health such as neurological conditions or symptoms (e.g., childhood seizures, autistic spectrum, head injury or concussion, history of transient ischaemic attacks, cerebrovascular disease, peripheral neuropathy) cardiovascular conditions of relevance (e.g., atrial fibrillation, uncontrolled diabetes, hypertension or hypercholesterolemia, sick-sinus syndrome, obstructive sleep apnoea, chronic obstructive pulmonary disease, history of cardiovascular surgery), metabolic dysfunctions (e.g., folate/vitamin B12 malabsorption, abnormal levels of thyroidstimulating hormone, lactose/gluten intolerance), ongoing pharmacological treatment with psychotropic or experimental 
TABLE 1 | Demographic and neuropsychological description of the sample.

\begin{tabular}{lccc}
\hline Variable & $\begin{array}{c}\text { Younger } \\
\text { Adults }\end{array}$ & $\begin{array}{c}\text { Older } \\
\text { Adults }\end{array}$ & $p$
\end{tabular}

\section{Demographic Indices}

$\begin{array}{lccr}\text { Age (years) } & 19.09(1.10) & 73.89(3.08) & <0.001 \\ \text { Education (years) } & 14.00(1.51) & 13.89(3.04) & 0.827 \\ \text { Sex (f/m) } & 26 / 19 & 28 / 17 & 0.667 \\ \text { Mini-Mental State Examination } & 29(2) & 29(2) & 0.987 \\ \text { Neuropsychological Assessment-Non-normally Distributed Tests } & \\ \text { Confrontation Naming Test } & 18(2) & 20(1) & <0.001 \\ \text { Paired Associated Learning Test } & 19(5) & 15(6) & <0.001 \\ \text { Pyramids and Palm Trees Test } & 49(3) & 51(2) & <0.001 \\ \text { Rey-Osterrieth Complex Figure Test-Copy } & 35(3) & 34(5) & 0.058 \\ \text { Rey-Osterrieth Complex Figure Test-Recall } & 22(7.275) & 16(9.5) & <0.001 \\ \text { Digit Span Test-Forward } & 7(2) & 7(3) & 0.983 \\ \text { Digit Span Test-Backward } & 5(2) & 5(3) & 0.244 \\ \text { Raven Coloured Progressive Matrices } & 33(4) & 33(3) & 0.381 \\ \text { Digit Cancellation Test } & 56(4) & 54(7) & 0.006 \\ \text { Visuoconstructive Apraxia Test } & 14(0) & 13(2) & <0.001 \\ \text { Stroop Test-Time Interference } & 10.3(6.07) & 21.5(13.1) & <0.001 \\ \text { Stroop Test-Error Interference } & 0(0) & 0(0) & 0.900 \\ \text { Token Test } & 34(1.5) & 35(2) & 0.122\end{array}$

Neuropsychological Assessment-Normally Distributed Tests

$\begin{array}{lccc}\text { WAIS-Similarities Test } & 20.31(4.46) & 22.56(6.60) & 0.063 \\ \text { Letter Fluency Test } & 39.02(10.32) & 45.56 & 0.021 \\ & & (15.53) & \end{array}$

Category Fluency Test-Normally Distributed Indices

\begin{tabular}{lccc} 
Test score: Two Categories & $33.80(6.65)$ & $33.69(7.02)$ & 0.939 \\
Category: Animals & $19.60(4.47)$ & $18.67(4.62)$ & 0.333 \\
Category: Fruits & $14.20(3.27)$ & $15.02(4.45)$ & 0.321 \\
Category Fluency Test-Non-normally Distributed Indices & & \\
Intrusions & $0(0)$ & $0(0)$ & 0.746 \\
Perseverations & $2(3)$ & $3(3)$ & 0.064 \\
\hline
\end{tabular}

"Age" and "Education" are typically normally distributed and are thus reported as means and standard deviations and analysed with t-tests.

"Sex" is indicated as frequency ratios and was analysed with a chi-square test. Scores on the Mini-Mental State Examination were not normally distributed and are thus indicated as medians and interquartile ranges and analysed with a MannWhitney $U$ test. Neuropsychological indices were also split into normally and non-normally distributed and reported as appropriate. Scores included in this table reflect uncorrected neuropsychological data.

medications, or with molecules with known toxic effects on internal organs, substance abuse, learning disabilities and presence of behavioural symptoms suggestive of underlying psychological dysfunction or difficulties (e.g., addiction, chronic anxiety/depression/apathy, mood or personality disorders, attention deficit hyperactivity disorder). Each volunteer was invited to the Department of Neuroscience at the University of Sheffield (United Kingdom) and completed a battery of neuropsychological tests. No participant had subjective cognitive complaints. Of the two groups, particular care was taken to evaluate diagnostic statuses in the group of older adults, since in this age range prevalence of cognitive impairment is estimated to range between 5\% and 40\% (Pais et al., 2020). To assess their cognitive profile the diagnostic labelling consensus proposed by the American Academy of Clinical Neuropsychology was followed, whereby performance above the expected 24th percentile is considered within normal limits (Guilmette et al., 2020). We thus used the entire cohort of $\geq 70$ year-old adults $(n=75)$ from which the study group of older adults had been extracted, to define numerical cut-offs corresponding to the 24th, 8 th, and 2 nd percentile for each test score. This was carried out to categorise performance into one of the following four labels: "score within normal limits", "low average score", "below average score", and "exceptionally low score" (Guilmette et al., 2020). For clinical interpretational purposes, we also relied on the principles outlined by Axelrod and Wall (2007) and by Binder et al. (2009), according to which a proportion of scores not within normal limits should be expected when a battery of tests is administered to healthy controls.

All participants provided their written informed consent prior to study inclusion. All procedures were carried out in compliance with the Declaration of Helsinki. The study was approved by the regional ethics committee of Yorkshire and Humber, reference number 05/Q1104/129.

\section{CFT-Scoring Procedures}

The "classic" 1-min version of the test was administered orally. Three categories were used: cities, animals and fruits (in this order). For the purposes of this study, only animals and fruits were analysed, since "cities" is a category based on the recall of proper nouns for which no linguistic ratings are available. Sub-scores on these two categories were modelled to evaluate cross-category consistency. Linear regression models were run to predict the number of correct "fruits" entries using the number of correct "animals" entries as predictor. This was carried out in the entire cohort and, separately, for each age group.

Each test performance was carefully reviewed and entries were scored as correct if they belonged to the target category (i.e., were not "intrusions") and if they were not "perseverations," (e.g., a repetition, a subordinate/superordinate to a word already produced such as "ape" and "gorilla", or the same entity in a different context such as "grape" and "raisin," or "sheep" and "lamb"). For a detailed description of these rules, please refer to the Supplementary Material. To ensure consistency in the scoring procedures across all 90 participants, a standardised form was defined for each entry that had been generated in multiple ways (e.g., "kiwi" and "kiwi fruit," or "hippo" and "hippopotamus"). Please consult the Supplementary Material for more details on standardised entries. All intrusions and perseverations were discarded. Post hoc analyses were, however, run on these data.

Each word was scored based on 17 item-level semantic and non-semantic descriptors: typicality, age of acquisition, concreteness, frequency, prevalence, recognition time, valence, arousal, dominance, body-object interaction, graphemes count, syllables count, consonant/vowel quantity ratio, phonological complexity, SRO, in-list orthographic Levenshtein distance, and dictionary orthographic Levenshtein distance. A description of these features (inclusive of examples) and the references from which linguistic ratings were obtained (Rosch, 1975; Murray and Forster, 2004; Yarkoni et al., 2008; Hargreaves et al., 2012; Kuperman et al., 2012; Warriner et al., 2013; Brysbaert 
et al., 2014, 2019; van Heuven et al., 2014; Dufau et al., 2015; Riley and Thompson, 2015; Räling et al., 2016; Pexman et al., 2019; Sohrabi, 2019; Mandera et al., 2020) are listed in Table 2.

\section{Feature-to-Feature Correlations}

Once scoring was completed for all items, the two categories (animals and fruits) were merged to maximise the size of individual data distributions. Coefficients of non-parametric correlation (Spearman's rho) were thus calculated to compute all 136 patterns of feature-to-feature association (Figure 2), i.e., $[(n \times(n-1)] / 2=136$. In case of missing data (i.e., words with no available rating for a specific feature), correlational models were run with the remaining available values. The count and proportional implications of missing data were reviewed throughout the cohort. Each participant had between 19 and 43 observations per each of the 17 features for the calculation of individual correlational profiles, with medians ranging between 30 (for valence, arousal, and dominance) and 33.5 (for typicality) observations. Only 16 of the 136 feature-to-feature correlations were analysed to comply with the first methodological approach (i.e., the correlation between SRO and the other 16 features; see Figure 3 for details on the 16 correlational patterns of interest), while the remaining 120 feature-to-feature correlations were not considered any further. These additional correlations, in fact, are unrelated to memory, but simply describe associations among pairs of semantic and non-semantic features (e.g., between "graphemes count" and "body-object interaction") that are of no direct interest to the study of SRO. To allow between-group inferential statistics, all coefficients were converted to $z$-scores, by applying a Fisher's rho-to-z transformation (Zar, 2005, Eq. 19).

All 16 distributions of feature-to-feature $z$-converted correlation coefficients were tested for normality (Shapiro-Wilk test), presence of outliers [the method recommended by Hoaglin, Iglewicz, and Tukey based on a $2.28 \times I Q R$ cut-off (Hoaglin et al., 1986)] and between-group homogeneity of variance (Levene's test). There were no missing data in these analyses.

\section{Graph-Theory Analysis of Correlations}

Commonly used in neuroimaging to analyse the complexity of brain networks (Bullmore and Sporns, 2009), graph theory is a mathematical framework that studies systems of variables related to each other in various (direct and indirect) ways. A graph is usually represented in the form of a schematic illustration in which variables are arranged in the two-dimensional space and connected to one another with a series of lines (Figure 2C). Variables are indicated as "nodes" of the graph while the word "edge" refers to a link that connects any two nodes on the basis of some established relationship. A third important concept is that of "neighbouring sub-graph" of a node ("NS", in the equations below), that is the set of nodes connected to it with an edge. Subject-specific graphs of 17 nodes were created and, to ensure that graphs included only significant node-to-node associations, the edge-forming rule was chosen based on the significance level of the correlation coefficients. To this end, two thresholds of significance were considered $(p<0.05$ and $p<0.01$ ). All edges were unweighted (i.e., having the same value) and undirected (i.e., expressing a significant, non-directional coefficient of correlation). Figures 2A-C illustrates an example of subject-specific graph, where edge-defining correlations were calculated in a dataset obtained from the administration of the CFT to a single individual.

Four metrics were calculated to characterise the node of interest (i.e., SRO): degree, betweenness centrality, global efficiency, and local efficiency. The arithmetical formula of each metric (Rubinov and Sporns, 2010) for a node " $i$ " is as follows (i.e., consult Figure 2D for a practical application of these four formulas on an individual CFT graph):

$$
\text { Degree }_{\mathrm{i}}=\sum N S_{i, j}
$$

The degree of a node is the sum of all edges linking it to other nodes (i.e., the number of significant correlations),

$$
\text { Betweenness Centrality }_{\mathrm{i}}=\frac{\sum_{j, k \neq i}\left[i \in P_{j, k}\right]}{(N-1)(N-2)}
$$

while its betweenness centrality is a fractional measure of the number of times the node is part of the shortest path (measured in number of edges; " $P$ " in the formula) that connects any two nodes of the graph (" $j$ " and " $k$ "). These two metrics were used as indices of direct centrality (degree) and global centrality, i.e., the central role played by nodes within the whole graph (betweenness centrality), respectively.

$$
\text { Global Efficiency } y_{\mathrm{i}}=\frac{\sum_{i \neq j} 1 / P_{i, j}}{N-1}
$$

Global efficiency of a node (an index of integration) is a proportion of the number of nodes of the graph and consists of the inverse of the average shortest path that links the node in question to the other nodes.

$$
\text { Local Efficiency } y_{\mathrm{i}}=\frac{\sum_{j \neq k \in N S_{i}} 1 / P_{j, k}}{d_{i}\left(d_{i}-1\right)}
$$

Local efficiency of a node is instead a proportion of the node's degree (" $d$ ", in the above formula) and consists of the inverse of the average shortest path between each pair of nodes that are part of the neighbouring sub-graph of interest (minus the node of interest itself).

To assess the performance of the two edge-forming rule candidates (i.e., correlations significant at a $p<0.05$ or 0.01 ), indices of cost efficiency were calculated (the cost of a node is equal to its degree divided by $\mathrm{N}-1$ ). These were not calculated for a single node (as with the formulas above) but for the entire graph (i.e., via an average of all nodal measures).

$$
\text { Cost Efficiency }=\text { Global Efficiency }- \text { Cost }
$$

A $p$-value $<0.05$ was associated with a significantly more convenient cost efficiency $\left(t_{89}=23.201, p<0.001\right.$; paired-sample $t$-test), and was thus retained as the edge-forming rule for this study. This choice resulted in a number of edges between 23 and 64 (out of 136) in the two cohorts (younger adults: mean $=43.71$, $S D=7.84$; older adults: mean $=46.58, S D=8.69$; there was no 
TABLE 2 | Description, inclusive of examples, of all 17 features included in this study.

\begin{tabular}{|c|c|c|c|}
\hline Feature & Description of Feature & Example (Category: Animals) & Reference for Normative Data \\
\hline \multicolumn{4}{|c|}{ Semantic Features } \\
\hline Typicality & $\begin{array}{l}\text { This feature reflects the "prototype approach" of } \\
\text { conceptual organisation, which posits that semantic } \\
\text { categories are organised based on an internal structure } \\
\text { (Rosch, 1975) and that each word is characterised by a } \\
\text { degree of semantic relatedness with other words of that } \\
\text { category (Räling et al., 2016). Within this structure, some } \\
\text { members of the category are more typical exemplars and }\end{array}$ & $\begin{array}{l}\text { OSTRICH: lower typicality (score = } \\
\text { 1.36); MOOSE: higher typicality } \\
\text { (score = 6.42). }\end{array}$ & $\begin{array}{l}\text { In-house normative data were } \\
\text { applied to score this feature: a } \\
\text { group of volunteers had been } \\
\text { asked to rate how representative a } \\
\text { word was of its own category, } \\
\text { assigning a score from } 1 \text { (least } \\
\text { typical) to } 7 \text { (most typical). }\end{array}$ \\
\hline
\end{tabular}

Age of Acquisition

Concreteness

Frequency

Prevalence

Recognition Time

Valence

Arousal

Dominance

Body-Object Interaction

Non-semantic Features

Graphemes Count

Syllables Count are recalled more promptly.

DUCK: earlier age of acquisition (estimated average: $\mathbf{3 . 5 3}$ years); CONDOR: later age of acquisition (estimated average: $\mathbf{1 3 . 0 8}$ years). system and solidify connections with other words than words acquired later in life. As a result, they are processed more rapidly and are more resistant to neural dysfunction (Sohrabi, 2019).

This feature (expressed as a number ranging from 1 to 5 ) was included as a control descriptor under the assumption that, to some extent, all animal and fruit words would be equally concrete. Although skewed towards a score of 5 , perceived concreteness of animal words was, possibly, in part "attenuated" by alternative meanings (e.g., MOLE, MANDARIN, to blow a RASPBERRY, etc.).

The frequency upon which each word appears in a certain language is significantly linked to how difficult/easy it is to access it from semantic memory (Murray and Forster, 2004). A 1-to-7 scale was used to quantify this feature.

This feature (expressed as z-converted percentages) indicates the proportion of people in a population who report they know the word in question, and captures aspects of word difficulty different from those tagged by other indices such as frequency or age of acquisition (Brysbaert et al., 2019).

This feature reflects the $z$-converted response time with which study participants indicated that they knew a specific word (Mandera et al., 2020). Recognition time is complementary to prevalence and provides fine-grained quantitative detail of inter-word variability.

This feature indicates the level of pleasantness evoked by the word. The score ranges from 1 to 9 .

This feature indicates the strength of the emotion induced by the word. The score ranges from 1 to 9 .

This feature indicates the level of perceived control towards the referent. The score ranges from 1 to 9 .

This feature (scored onto a scale from 1 to 7 ) quantifies the possibility offered by the referent of a word to be interacted with. It is a semantic quality that embodies the sensorimotor information associated with a certain word (Hargreaves et al., 2012).

The orthographic transcription of the word was scored. Spaces separating two terms (e.g., as in "GUINEA PIG" or "PASSION FRUIT") were not counted.

Although strongly correlated with the number of graphemes, this feature was included as there are examples of common words in which this correspondence is invalid.
THRUSH: lower concreteness (score = 3.92); WALRUS: maximum concreteness (score $=\mathbf{5 . 0 0}$ ).

MANATEE: lower frequency (score = 2.08); FISH: higher frequency (score $=5.19$ ).

DORMOUSE: lower prevalence (score = 0.31); SLOTH: higher prevalence (score $=\mathbf{2 . 5 8}$ ).

SPIDER: faster recognition (score = -0.69); ANTEATER: slower recognition (score $\mathbf{= 0 . 1 0}$ ).

WASP: lower valence (score = 2.71); PANDA: higher valence (score $=7.55$ ).

SEAL: lower arousal (score $=\mathbf{2 . 5 0}$ ); CROCODILE: higher arousal (score $=6.48$ ).

BEAR: lower dominance (score = 3.59); BULL: higher dominance (score = 6.89).

PLATYPUS: lower body-object interaction (score = 3.04); DOG: higher body-object interaction (score $=6.40$ ).

OX: shorter word (2 graphemes); CATERPILLAR: longer word (11 graphemes).

IGUANA: 3 syllables (with 6 graphemes); SHRIMP: 1 syllable (with 6 graphemes).
Kuperman et al., 2012

Brysbaert et al., 2014

The SUBTLEX database for British English (van Heuven et al., 2014).

The English Crowdsourcing Project, an internet-based initiative in which native English speakers were asked to indicate whether they knew a certain word or not (Mandera et al., 2020).

As with prevalence, the English Crowdsourcing Project (Mandera et al., 2020).

Warriner et al., 2013; although pleasantness of words is a subjective trait, rating dispersion was relatively low.

Warriner et al., 2013

Warriner et al., 2013

Pexman et al., 2019

N/A 
TABLE 2 | Continued

\begin{tabular}{|c|c|c|c|}
\hline Feature & Description of Feature & Example (Category: Animals) & Reference for Normative Data \\
\hline $\begin{array}{l}\text { Consonant/Vowel } \\
\text { Quantity Ratio }\end{array}$ & $\begin{array}{l}\text { This feature, meant to capture the ratio of consonant and } \\
\text { vowel quantity, represents a basic phonological } \\
\text { descriptor expected to be completely unrelated to the } \\
\text { difficulty of word retrieval. The scoring was carried out on } \\
\text { the UK phonetic transcription of the word. }\end{array}$ & $\begin{array}{l}\text { BUFFALO ("b } \Lambda \text { fələひ"): } 7 \text { phonemes } \\
3 \text { of which are consonants }=\mathbf{0 . 4 3} \text {. }\end{array}$ & Dufau et al., 2015 \\
\hline $\begin{array}{l}\text { Phonological } \\
\text { Complexity }\end{array}$ & $\begin{array}{l}\text { Complexity of consonant clusters was scored based on } \\
\text { the UK phonetic transcription of the word, following the } \\
\text { model of consonant sonority and scoring proposed by } \\
\text { Riley and Thompson (2015). As word length may } \\
\text { influence this feature (i.e., the longer the word, the more } \\
\text { consonants there may be), the additive complexity score } \\
\text { of all clusters within a word was partialised by the number } \\
\text { of syllables. }\end{array}$ & $\begin{array}{l}\text { PHEASANT ("fezənt"): } 3 \text { consonant } \\
\text { clusters. 1) "f", voiceless fricative, } \\
\text { sonority of } 5 ; 2 \text { ) "z", voiced fricative, } \\
\text { sonority of } 4 ; 3 \text { ) "nt": combination } \\
\text { of a nasal occlusive, sonority of } 3 \text {, } \\
\text { and a voiceless stop, sonority of } 7 \text { : } \\
\text { combined sonority of } 4 \text {. Global } \\
\text { score }=13 \text {. Partialised score (2 } \\
\text { syllables) }=\mathbf{6 . 5} \text {. }\end{array}$ & Riley and Thompson, 2015 \\
\hline Serial Recall Order & $\begin{array}{l}\text { An incremental score from } 1 \text { to } n \text { was assigned to each } \\
\text { correct entry (from the first to the last) generated for each } \\
\text { category. This variable reflects the serial order with which } \\
\text { words are recalled via the semantic cue assigned and is } \\
\text { expressed as an ordinal scale. }\end{array}$ & $\begin{array}{l}\text { e.g., CAT (1), DOG (2), HORSE (3), } \\
\text { SHEEP (4), DUCK (5), SWAN (6), } \\
\text { LION (7), TIGER (8), GIRAFFE (9) } \\
\text {.. }\end{array}$ & N/A \\
\hline $\begin{array}{l}\text { In-List Orthographic } \\
\text { Levensthein Distance }\end{array}$ & $\begin{array}{l}\text { This feature is a metric of similarity between two } \\
\text { orthographic strings (Yarkoni et al., 2008). Each word was } \\
\text { compared to every other word generated by the } \\
\text { participant to obtain word-to-word distances based on } \\
\text { the minimum number of graphemes that would need to } \\
\text { be replaced/removed/inserted. An average distance was } \\
\text { then calculated for each word in relation to all other } \\
\text { words. }\end{array}$ & $\begin{array}{l}\text { PARROT (target word); } \text { HORNET } \\
\text { (comparison word 1): distance = 4; } \\
\text { PANTHER (comparison word 2): } \\
\text { distance = 5; OCELOT (comparison } \\
\text { word 3): distance = 4; average } \\
\text { distance = 4.33. Underlined are the } \\
\text { elements of difference that } \\
\text { constitute the distances. }\end{array}$ & $\begin{array}{l}\text { Scoring was carried out through the } \\
\text { resources provided at https://www. } \\
\text { dcode.fr/levenshtein-distance. }\end{array}$ \\
\hline $\begin{array}{l}\text { Dictionary Orthographic } \\
\text { Levensthein Distance }\end{array}$ & $\begin{array}{l}\text { This feature is a metric of the 'orthographic } \\
\text { neighbourhood' of a word. Levensthein Distances were } \\
\text { calculated to establish the number of terms in the entire } \\
\text { English dictionary differing from the target word by one } \\
\text { grapheme. }\end{array}$ & $\begin{array}{l}\text { OTTER (target word); number of } \\
\text { words that differ by one grapheme } \\
=7: \underline{\text { UTTER}} \text { OTTERS, } \underline{\text { HOTTER, }} \\
\text { POTTER, OUTER, OTHER, } \\
\underline{\text { COTTER. Underlined are the }} \\
\text { elements of difference that } \\
\text { constitute the distances. }\end{array}$ & $\begin{array}{l}\text { As with the previous feature, } \\
\text { scoring was carried out via the } \\
\text { resources provided at https://www. } \\
\text { dcode.fr/levenshtein-distance. }\end{array}$ \\
\hline
\end{tabular}

between-group difference). The calculation of these indices was carried out using the Brain Connectivity Toolbox ${ }^{1}$, implemented in MATLAB (R2014a, Mathworks Inc., United Kingdom).

\section{Statistical Inference}

To address the first hypothesis, coefficients of correlation (Spearman's rho) were run to test the association between standard and correlational CFT indices of interest and two measures selected from the neuropsychological battery: the Digit Cancellation Test (Della Sala et al., 1992) as a measure of processing speed and the Stroop Test-Time Interference (Venneri et al., 1992) as a measure of executive functioning. A conservative $p$-value $<0.01$ was used as statistical threshold.

To address the second hypothesis, one-way analyses of covariance (ANCOVAs) were run to compare the correlational profiles of younger and older adults. Both $z$-transformed correlation coefficients and graph metrics were analysed. Each model was corrected for years of education as a proxy of cognitive reserve (Stern, 2009), Mini-Mental State Examination score (Folstein et al., 1975) as an index of overall cognitive

\footnotetext{
${ }^{1}$ https://sites.google.com/site/bctnet/Home/functions
}

functioning and raw CFT score to control for the variability in the number of entries at the basis of the correlation. These were all included as covariates. As above, a conservative $p$-value $<0.01$ was used as statistical threshold in the analyses of $z$-transformed coefficients of correlation. Given the novelty and the exploratory nature of the graph-metrics approach, a more lenient $p$-value of 0.05 was instead used as threshold of significance in the analysis of graph theory metrics.

\section{RESULTS}

The application of study criteria resulted in the recruitment of 250 healthy controls resident in the United Kingdom Yorkshire and Humber region, including 45 younger adults aged 1821 years old (who were all entered in this study) and 75 older adults aged $\geq 70$ years old, 45 of whom were randomly selected for this investigation. The demographic and cognitive profile of the two groups is included in Table 1. All participants were monolingual English native speakers of White-British ethnicity who were born and had their educational training in the United Kingdom. They all took part in the data collection on a voluntary basis and received no compensation or academic credits in return. 
A

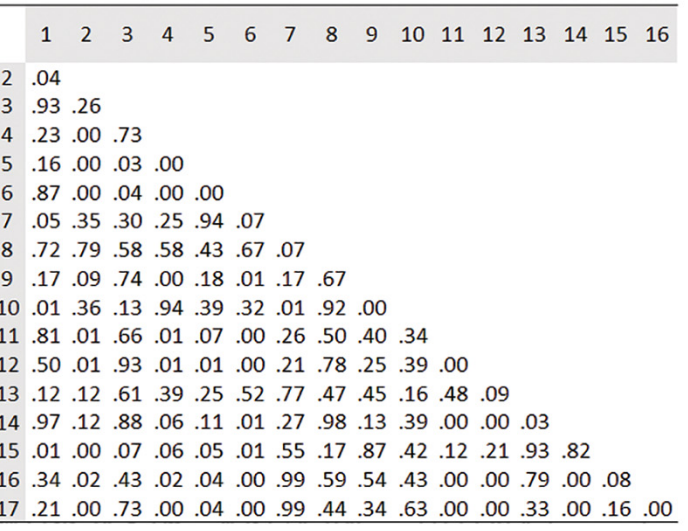

B

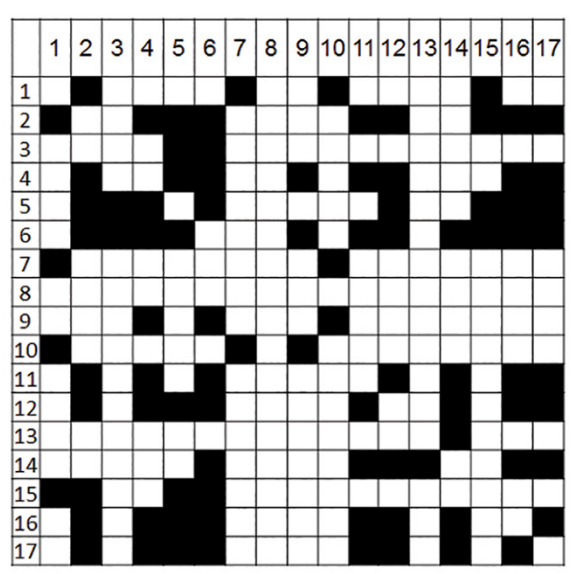

C

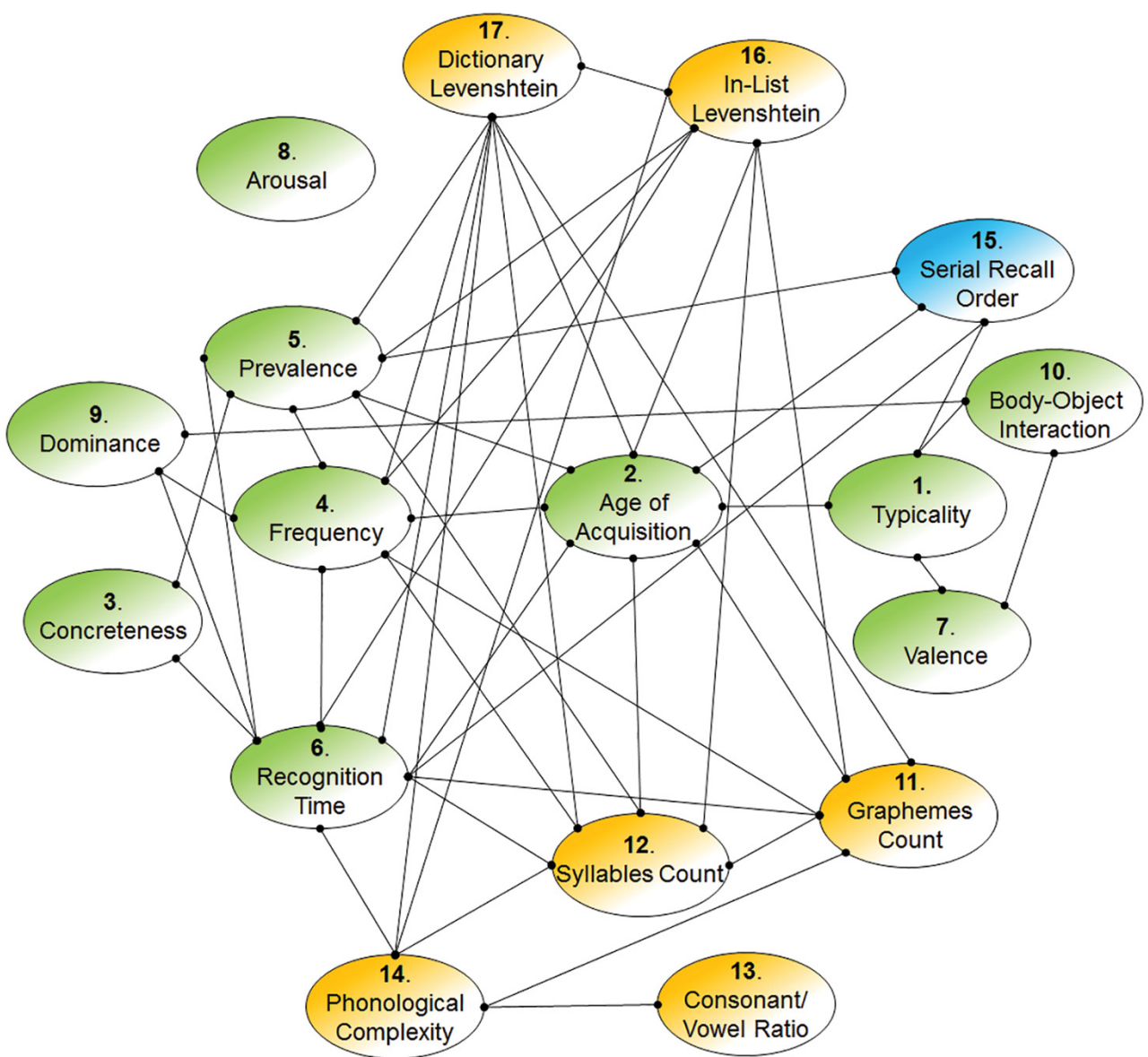

D

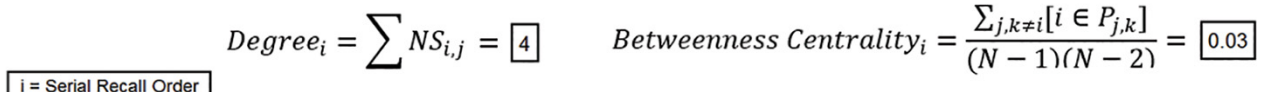

$$
\begin{aligned}
& \text { Global Efficiency }=\frac{\sum_{i \neq j} 1 / P_{i, j}}{N-1}=0.5833 \quad \text { Local Efficiency } i=\frac{\sum_{j \neq k \in N S_{i}} 1 / P_{j, k}}{d_{i}\left(d_{i}-1\right)} 0.8333
\end{aligned}
$$

FIGURE 2 | Example of matrices and graph calculated on a single participant (a 71 year-old woman). The feature-to-feature correlational matrix (A) and the binary adjacency matrix tagging significant correlations (B) are shown. Please note that, since based on correlations, adjacency matrices express bidirectional relationships. The graph (C) colour-codes and distinguishes the node of interest (blue) from the ten semantic features (green) and the other non-semantic features (yellow). Nodal metrics of "serial recall order" (inclusive of formulas) for this specific participant are reported in the lower part of the image (D). 


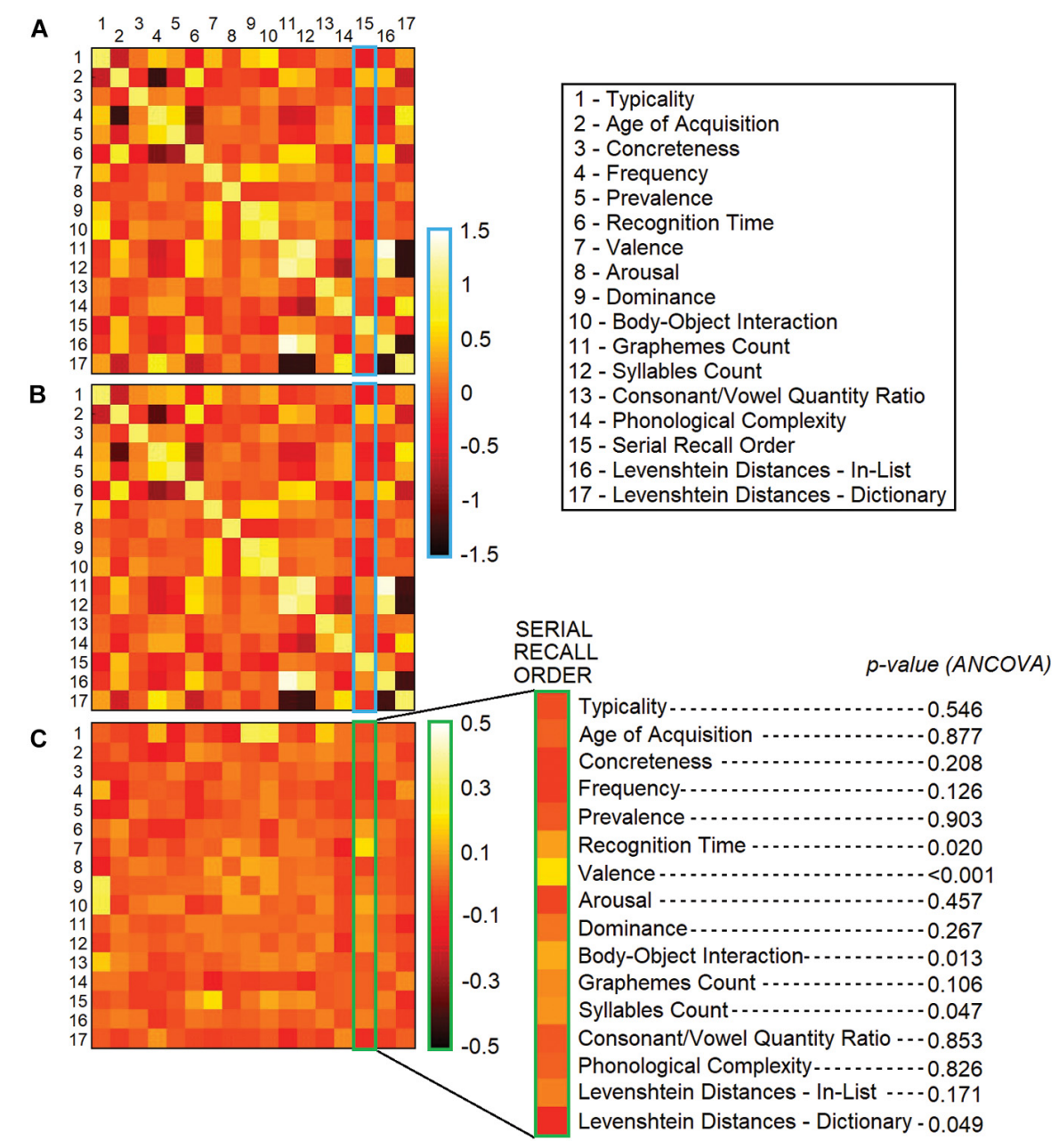

FIGURE 3 | z-transformed coefficients of correlation calculated across the entire $17 \times 17$ matrix within the group of older adults (A) and younger adults (B). Between-group difference scores (where scores among older and younger adults are the subtrahend and minuend, respectively) are shown below (C), flanked by the outcome of statistical comparisons. Blue and green frames were added to highlight the coefficients of correlation relevant to this study.

\section{Cognitive Profiles}

The classification of test performance carried out in the group of older adults using the framework by Guilmette and coauthors (Guilmette et al., 2020) revealed that the majority $(\sim 85 \%)$ of test scores was "within normal limits," with a further $\sim 10 \%$ of "low average," $\sim 5 \%$ "below low average" and less than $1 \%$ "exceptionally low" scores. This was consistent with rates expected in healthy controls assessed with a multi-test battery (Axelrod and Wall, 2007; Binder et al., 2009). In addition, none of the participants met the criteria for a diagnosis of mild cognitive impairment. Table 1 illustrates the cognitive profiles of the two groups. Younger adults performed significantly better on tests of long-term episodic memory (Paired Associated Learning Test and the recall of the Rey-Osterrieth Complex Figure), visuo-constructive abilities (Visuoconstructive Apraxia Test and the copy of the Rey-Osterrieth Complex Figure) and attentive/inhibitory skills (Digit Cancellation Test and Stroop Test time interference), while older adults scored significantly better on tests measuring lexical/semantic processing and
SM (Letter Fluency Test, Confrontational Naming Test, and Pyramids and Palm Trees Test). These group differences are in line with the trends commonly seen in association with normal ageing. Performance on the Stroop test (arguably the task in the battery with the highest cognitive demands) indicated time-interference latencies $<46.5 \mathrm{~s}$ and $<25 \mathrm{~s}$ in the group of older and younger adults, respectively, suggesting satisfactory levels of commitment during task performance. In addition, as performance on the Raven's Coloured Progressive Matrices is often used as a proxy of general non-verbal IQ (Wongupparaj et al., 2015), an inspection of scores on this test indicated normal intelligence in all participants.

In total, 3311 words were generated by the entire cohort as part of the CFT, including 254 (7.7\%) perseverations and $20(0.6 \%)$ intrusions. No group differences on the CFT were found either when "animals" and "fruits" were analysed separately, or when they were combined. The analyses of cross-category consistency revealed a significant linear association across the whole cohort, with valid "animals" entries significantly predicting the number 
of valid "fruits" $(b=0.339)$. Trends in the same direction were found when analyses were run separately in each age group, with older adults showing a weaker association $(b=0.205)$ and younger adults showing a stronger association $(b=0.634)$. A visual representation of these linear associations and the results of a validation analysis carried out in an independent cohort are reported in the Supplementary Material.

\section{Feature-to-Feature Correlations}

Fifteen out of 16 distributions of feature-to-feature correlational scores met the assumptions of normality. The only distribution in breach of the assumption was that of the $z$-converted correlation coefficient between SRO and age of acquisition. This was also the only distribution in which an outlier (an older adult) was detected. After removing the outlier, the assumption was met. In addition, between-group homogeneity of variance was confirmed for all but three correlational features: those between SRO and concreteness, prevalence and dictionary orthographic Levenshtein distance. In all three cases older adults had a wider distribution with a total of five extreme values located at a $>1.5 \times I Q R$ distance from the upper/lower quartile. After removing these five data-points, the assumption was met.

The standard CFT score was significantly correlated with performance on the Digit Cancellation Test ( $r h o=0.279$, $p=0.002$ ). None of the SRO-based correlations was associated with performance on the Digit Cancellation Test or Stroop TestTime Interference.

The direction of the association (i.e., the sign of the correlation coefficient) was the same in both groups for all 16 models. Only one standardised correlation coefficient out of the pool of 16 differed between the two groups, i.e., that between SRO and valence $\left(F_{1,85}=15.979, p=0.00014, \eta_{p}^{2}=0.158\right.$; Figure 3$)$. This association was still significant even when the analysis was corrected for all other $15 z$-transformed correlation coefficients, included as covariates $\left(F_{1,70}=14.255, p=0.00033, \eta^{2}{ }_{p}=0.172\right)$. As words were recalled, the decrease in valence was steeper in younger adults. To characterise this pattern more in detail, words retrieved in positions $1-5,6-10,11-15$, and 16-20 were grouped together for post hoc analysis. ANOVA models were thus designed to test the effect of age group on each positional set, controlling for years of education and Mini-Mental State Examination score (the raw CFT score was not included as a covariate in these models as it is a property of the entire 1-min performance and is unrelated to the words generated in each positional set). Only words in position 1-5 differed between the two age groups, with younger adults retrieving words of significantly higher valence $\left(p<0.001, \eta^{2} p=0.122\right.$; Figure 4). The words most commonly generated by the two groups in position 1-5 are reported in Table 3. When positional sets were analysed for each separate category, animals $1-5$ showed a significant difference $(p=0.004$, $\left.\eta_{p}^{2}=0.094\right)$ while only a trend was observed for fruits $1-5$.

\section{Graph-Theory Analysis}

Nodal properties of SRO were extracted from each subjectspecific graph for the purpose of group-level analyses. Edge frequency in the two groups is illustrated in Figure 5. The SRO node counted a total of 431 edges across the whole cohort (older adults: 239, younger adults: 192), 318 of which ( 74\%) were toward a semantic node. The five nodes most often correlated (and thus expressing an edge) with SRO were typicality (61 times out of 90), age of acquisition (52 times), body-object interaction ( 47 times), frequency ( 46 times) and recognition time (34 times). The five least frequently correlated nodes were instead consonant/vowel quantity ratio (4 times), arousal (6 times), concreteness (8 times), phonological complexity (11 times), and dominance (15 times). A series of chi-square tests were run to compare edge frequency between the two groups. Older adults had more edges between SRO and recognition time $(\varphi=0.229)$, graphemes count $(\varphi=0.223)$, syllables count $(\varphi=0.255)$ and the orthographic Levenshtein distance between words and dictionary entries $(\varphi=0.236)$; all $p$-values $<0.05$.

Statistical differences for the node of interest between the two groups were found in two of the four metrics: degree and betweenness centrality (Table 4A). SRO was characterised by significantly lower betweenness centrality $\left(F_{1,85}=4.002\right.$, $\left.p=0.049, \eta_{p}^{2}=0.045\right)$ and by higher degree $\left(F_{1,85}=4.323\right.$, $\left.p=0.041, \eta_{p}^{2}=0.048\right)$ in the group of older adults. Younger adults had an average of 4.24 edges connecting SRO to other nodes, while older adults had an average of 5.29. The count of the edges from SRO toward semantic nodes, however, was similar between groups (older adults: mean $=3.64, S D=1.57$; younger adults: mean $=3.44, S D=2.00)$. Metric-to-metric correlation coefficients (Pearson's $r$ ) are reported in Table $4 \mathbf{B}$.

\section{Link Between Significant Metrics and Cognitive/Demographic Variables}

To explore the association between the 20 metrics investigated in this study (16 feature-to-feature $z$-transformed correlations and four nodal graph-theory metrics) and performance on standard cognitive tests (those included in the "Neuropsychological Assessment" sections of Table 1, other than Digit Cancellation Test and Stroop Test-Time Interference), coefficients of correlation were calculated at post hoc within the entire group of 90 adults using a Bonferroni-corrected $p<0.0025(0.05 / 20)$ and controlling each model for the same covariates as in the main analyses (Spearman's coefficient of partial non-parametric correlation). One sole correlation retained statistical significance: the $z$-transformed coefficient of correlation between SRO and valence was significantly correlated with performance on the Pyramids and Palm Trees test $\left(r h o_{85}=0.333, p=0.002\right)$. Associations significant at an uncorrected, more lenient $p<0.05$ are illustrated in the Supplementary Material.

We also tested the association between the 20 outcome metrics and the number of intrusions and perseverations made by participants during CFT. No model was significant at a Bonferroni-corrected $p<0.0025$. Associations significant at an uncorrected, more lenient $p<0.05$ are illustrated in the Supplementary Material.

Finally, we tested the association between the 20 outcome metrics and three major demographic variables: education, Mini-Mental State Examination score and sex, using the same threshold of significance. Education was significantly correlated with the $z$-transformed coefficient of correlation between SRO 

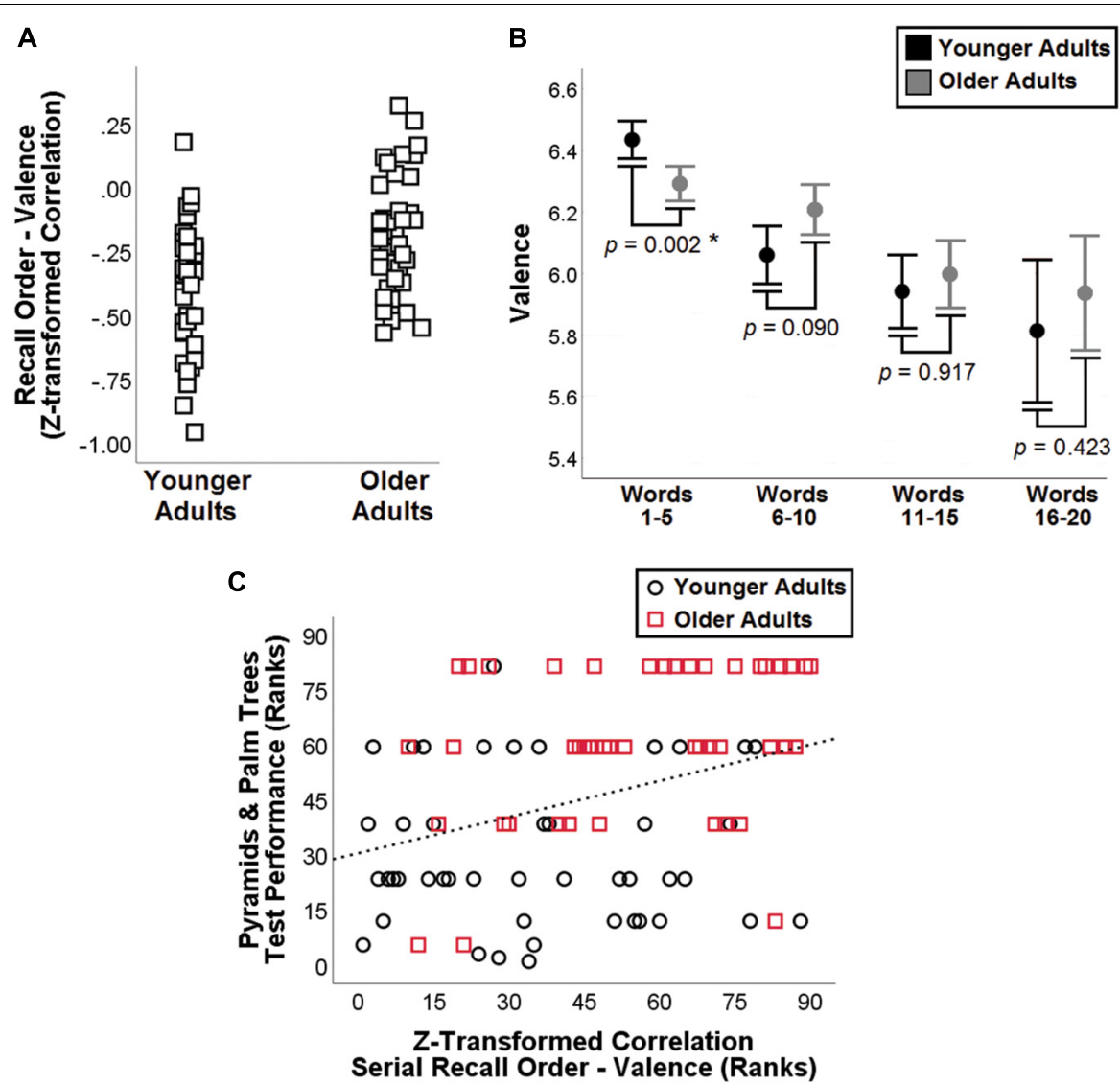

FIGURE 4 | Outcome of feature-to-feature correlation analysis. Group distributions of the z-transformed coefficient of correlation between serial recall order and valence is shown on the left (A), while post hoc analyses of five-word positions are shown on the right (B). The association between ranked z-transformed correlation coefficients and performance on the Pyramids and Palm Trees Test is shown below (C).

and Graphemes count $\left(r_{90}=-0.344, p=0.001\right)$, while general cognitive functioning measured via the Mini-Mental Examination Score was significantly correlated with two nodal indices of graph theory: SRO degree $\left(r h o_{90}=0.323, p=0.002\right)$ and SRO global efficiency (rho $90=0.321 p=0.002)$. As sex had a binary distribution, differences between males and females were tested with $t$-tests. No between-group differences, however, emerged as significant. Associations significant at an uncorrected, more lenient $p<0.05$ are illustrated in the Supplementary Material.

\section{DISCUSSION}

The study of SM is of particular interest to cognitive neuroscientists. There is, however, a methodological need for fine-grained measures of SM that are not excessively influenced by other functions. The CFT is often chosen by clinicians and researchers as preferred test of SM because, compared to other instruments (e.g., Boston Naming Test, Pyramids and Palm Trees/Camel and Cactus Test, the "Similarities" subtest of the Wechsler Adult Intelligence Scale, or tests based on recognition of famous people), it is a measure of free recall
(Gruenewald and Lockhead, 1980) and does not require any adaptation for cross-cultural or cross-linguistic use. Differently from cued recall and recognition, free recall is a self-initiated form of retrieval more aligned with real-life scenarios (Craik, 1983), and this confers a degree of ecological validity to this mode of testing. The CFT is also methodologically convenient, since it is simple and quick to administer and does not require a complex set-up. Moreover, it can be transposed into any language without requiring complex translations or validation studies. Facilitated by these aspects, it has proven to be a particularly versatile test, since a considerable number of innovative scoring procedures have been put forward, in an attempt to improve and optimise test measures that can be of assistance in clinical practice. In line with this goal, in this study we have devised a scoring method that combines the serial order of CFT word retrieval with the semantic "difficulty" of each word, quantified as a function of 16 separate semantic and non-semantic features. To put the validity of this profile of correlational variables to the test, we formulated a first hypothesis based on which correlational indices linking SRO to semantic features would be less statistically associated with performance on tests of speed of processing and executive functioning (functions that are known to support CFT performance) than the standard CFT score. We 
TABLE 3 | Words generated by each group in position 1-5.

\begin{tabular}{|c|c|c|c|c|c|c|c|}
\hline \multicolumn{4}{|c|}{ Younger Adults } & \multicolumn{4}{|c|}{ Older Adults } \\
\hline Word & Count & $\begin{array}{c}\text { Valence: Global Normative } \\
\text { Score (Younger Adults Score) }\end{array}$ & $\begin{array}{c}\text { Age } \\
\text { Difference }\end{array}$ & Word & Count & $\begin{array}{l}\text { Valence: Global Normative } \\
\text { Score (Older Adults Score) }\end{array}$ & $\begin{array}{c}\text { Age } \\
\text { Difference }\end{array}$ \\
\hline \multicolumn{8}{|l|}{ Animals } \\
\hline DOG & 39 & $7.00(7.09)$ & -0.09 & DOG & 33 & $7.00(6.89)$ & 0.11 \\
\hline CAT & 38 & 6.95 (6.50) & 0.45 & CAT & 31 & $6.95(7.40)$ & -0.45 \\
\hline LION & 12 & $5.84(6.10)$ & -0.26 & cow & 17 & $5.42(5.40)$ & 0.02 \\
\hline MOUSE & 12 & $4.80(4.75)$ & 0.05 & HORSE & 12 & $6.05(6.21)$ & -0.16 \\
\hline $\mathrm{FISH}$ & 10 & $6.42(6.43)$ & -0.01 & MOUSE & 12 & $4.80(4.83)$ & -0.03 \\
\hline HAMSTER & 10 & $5.88(6.44)$ & -0.56 & $\mathrm{PIG}$ & 12 & $4.83(4.78)$ & 0.05 \\
\hline HORSE & 10 & $6.05(5.83)$ & 0.22 & SHEEP & 12 & $5.32(5.10)$ & 0.22 \\
\hline RABBIT & 10 & $7.21(6.89)$ & 0.32 & LION & 10 & $5.84(5.56)$ & 0.28 \\
\hline BEAR & 8 & $5.33(5.36)$ & -0.03 & GOAT & 8 & $5.30(5.10)$ & 0.20 \\
\hline ELEPHANT & 8 & $6.17(5.57)$ & -0.40 & RABBIT & 7 & $7.21(7.50)$ & -0.29 \\
\hline TIGER & 8 & $6.00(6.64)$ & -0.64 & ELEPHANT & 6 & $6.17(6.55)$ & -0.38 \\
\hline GIRAFFE & 7 & $6.52(6.00)$ & 0.52 & RAT & 6 & $3.21(2.69)$ & 0.52 \\
\hline RAT & 6 & $3.21(3.45)$ & -0.24 & TIGER & 5 & $6.00(5.36)$ & 0.64 \\
\hline SHEEP & 6 & $5.32(5.56)$ & -0.24 & cow & 5 & $5.42(5.44)$ & -0.02 \\
\hline PIG & 5 & $4.83(4.89)$ & -0.06 & & & & \\
\hline \multicolumn{8}{|l|}{ Fruits } \\
\hline APPLE & 44 & $6.62(7.25)$ & -0.63 & APPLE & 40 & $6.62(6.47)$ & 0.15 \\
\hline BANANA & 34 & $6.71(6.56)$ & 0.15 & ORANGE & 34 & $6.81(7.00)$ & -0.19 \\
\hline PEAR & 33 & $6.70(6.80)$ & -0.10 & PEAR & 29 & $6.70(6.60)$ & 0.10 \\
\hline ORANGE & 29 & $6.81(6.43)$ & 0.38 & BANANA & 25 & $6.71(7.20)$ & -0.49 \\
\hline GRAPE & 14 & $6.70(6.27)$ & 0.43 & GRAPE & 9 & $6.70(7.22)$ & -0.52 \\
\hline KIWI & 8 & $6.11(6.50)$ & -0.39 & LEMON & 9 & $6.37(6.20)$ & 0.17 \\
\hline PINEAPPLE & 8 & $6.90(6.33)$ & 0.57 & GRAPEFRUIT & 7 & $5.77(6.00)$ & -0.23 \\
\hline STRAWBERRY & 8 & $7.25(6.91)$ & 0.34 & PEACH & 6 & $6.83(7.20)$ & -0.37 \\
\hline MANGO & 6 & $6.57(7.75)$ & -1.18 & MELON & 5 & $6.32(6.23)$ & 0.09 \\
\hline PEACH & 5 & $6.83(6.38)$ & 0.45 & PLUM & 5 & $6.15(6.20)$ & -0.05 \\
\hline TOMATO & 5 & $5.80(5.00)$ & 0.80 & & & & \\
\hline
\end{tabular}

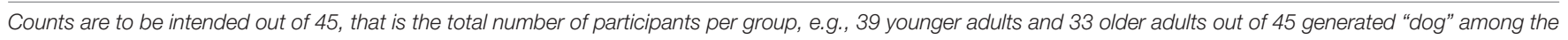
first five recall positions. Frequencies of 4 and less are not shown.

then formulated a second hypothesis addressing the effect normal ageing has on SM, with the expectation of a pattern of results aligned with older adults showing a more robust profile. To do so, we analysed the differences between younger and older adults, modelling $z$-transformed correlation coefficients in a direct way and indirectly, via the calculation of graph-theory metrics.

Although coefficients were similar between the two groups, the SRO-valence correlation indicated a robust difference (significant at a $p<0.001)$. Post hoc analyses showed that in the initial portion of the test (i.e., the first five words), older adults generated words of lower valence (i.e., typically perceived as less pleasant) than those generated by younger adults. While both age groups showed an overall decrement in valence as more words were generated, this decrease was steeper in the group of younger adults, as indicated by a significantly stronger coefficient of negative correlation. Experimental evidence indicates that there is a close relationship between SM and valence attribution (Bertoux et al., 2020). Other than showing consolidated semanticmemory skills (Nyberg et al., 1996, 2003; Park et al., 2002; Verhaeghen, 2003; Rönnlund et al., 2005; Small et al., 2011), older adults also show an "age-related positivity" effect, whereby stimuli of positive value have a processing advantage over stimuli of negative value (Reed and Carstensen, 2012). The combination of better SM and better processing of positive items indicates that older adults may be naturally prone to relying on valence during CFT performance. A similar trait does not characterise performance of younger adults, who show instead high level of valence only at the start of their performance (i.e., positions 1-5), when words are recalled with a high degree of automaticity and with limited need of semantic-control resources (Hurks et al., 2004) or strategies. We then tested whether age might play a role in the perceived valence of words. Evidence indicates that age is a significant, yet modest-at-best predictor of attributed valence, with $\eta_{p}^{2}$ effect sizes ranging from 0.001 (Söderholm et al., 2013) to 0.03 (Grühn and Smith, 2008), to 0.06 (Gilet et al., 2012), to an inferable Cohen's $d$ of 0.036 (Warriner et al., 2013). Our finding, however, cannot be ascribed to age differences in assigned valence because we relied on ageindependent ratings, i.e., the same ratings were used for both groups (Warriner et al., 2013). We propose, therefore, that age 


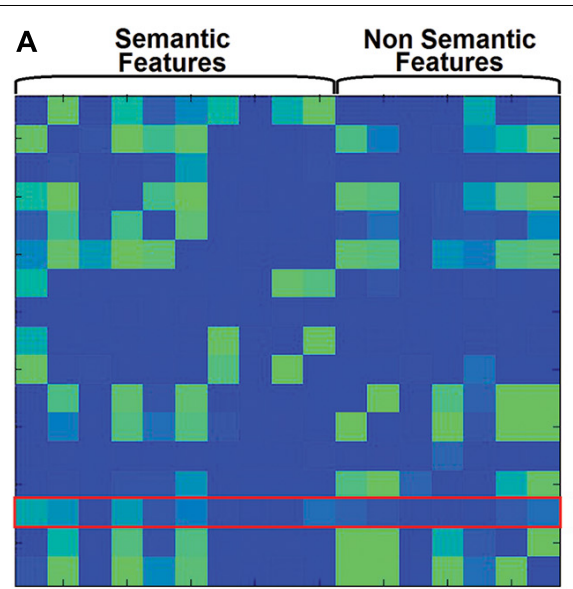

Older Adults

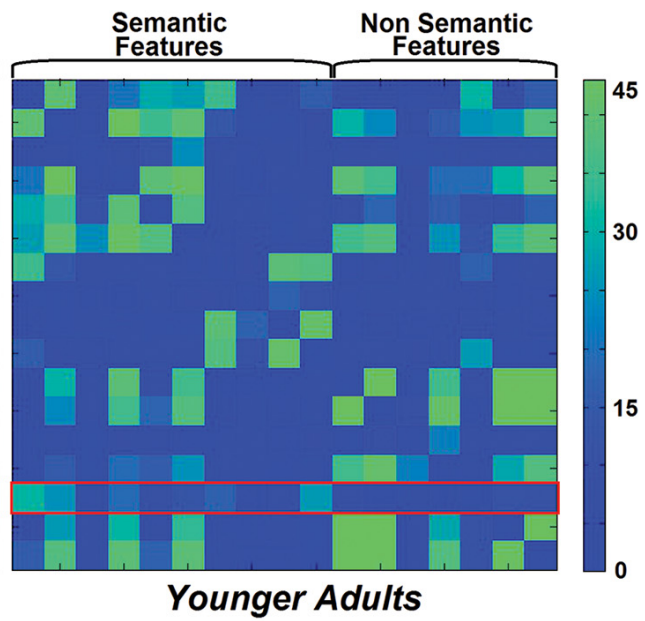

Younger Adults

B

Older Adults vs. Younger Adults - Comparison

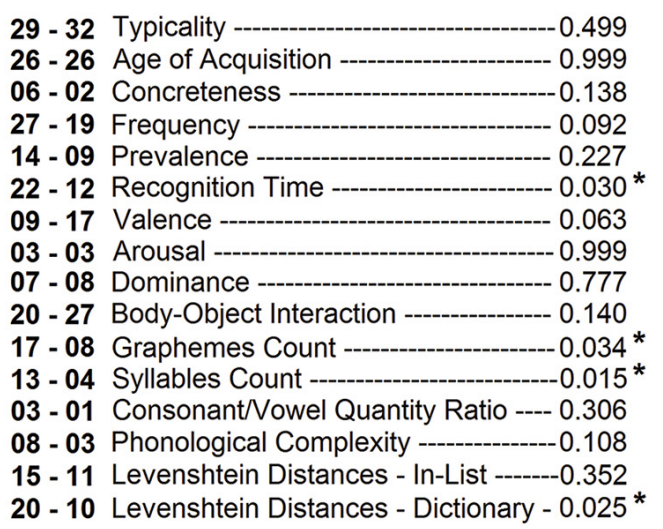

FIGURE 5 | Edge frequency in the two groups. A red frame was added to highlight the edges relevant to this study (A). A count of all these edges within each group is included below together with the outcome of the chi-square tests comparing edges between the two group frequencies, older and younger adults, respectively (B). Four pathways showed significant between-group differences. These same pathways approached or showed a trend toward significance when z-transformed correlation coefficients were analysed, as illustrated in Figure 2. Similarly, the edge toward valence approached significance in these analyses.

differences exist in the degree to which automatic semantic retrieval is susceptible to pleasantness-related effects. There is experimental evidence that retrieval from memory is influenced by valence. The findings of an experiment carried out with younger adults showed that immediate recall of pleasant words is higher than immediate recall of neutral words (Monnier and Syssau, 2008). The representation of words with a positive or negative valence is semantically richer than that of neutral words, and pleasant words in particular also embed a "life-enhancing" connotation, enabling "stronger semantic relatedness" (Majerus and D'Argembeau, 2011, p. 182). This signifies that automatic semantic processing elicited by CFT in younger adults would tend to rely more on such "hedonistic" aspect. Although a precise explanation of the neural mechanisms that underpin this difference is beyond the scope of this study, research has highlighted that, differently from controlled elaboration of emotional content, automatic emotional processing of word stimuli involves the left hemisphere more than the right hemisphere (Abbassi et al., 2011). Functional asymmetries are typical of neurological processing and ageing is known to be associated with processes of dedifferentiation (Koen and Rugg, 2019), asymmetry reduction and recruitment of additional regions in support of task performance (Berlingeri et al., 2013). If lateralised specialisation during automatic verbal emotional processing is attenuated by age, this could play a pivotal role in accounting for the sharp difference in valence observed between the two groups in the first five-word interval. Nonetheless, older adults perform at the same level as younger adults without exploiting any valence-related boost at the start of the task. This may indicate optimised retrieval from SM that does not "impetuously" rely on a prominent feature that is limited to a short-lived effect. In support of the interpretation that more neutral and "stable" valence is indicative of better function, we found a positive correlation (the less steep the decline, the better the performance) between the $z$-transformed valence coefficient and performance on the Pyramids and Palm Trees Test, a nonverbal measure of SM unaffected by processing speed and with limited executive demands. 
We acknowledge, however, that other, non-neurological factors might be at play. A close inspection of words retrieved in position 1-5 (Table 3) indicates that older adults retrieved more farm animals (i.e., cow, horse, pig, sheep, and goat were recalled 61 times by older adults and 34 times by younger adults) and fewer fruits typically considered "exotic" in the United Kingdom (i.e., banana, kiwi, pineapple, mango, coconut, and papaya were recalled, in total, 34 times by older adults and 58 times by younger adults). It is known that early sociocontextual exposures influence cognitive functioning in later life (Meyer et al., 2020). On these grounds, people in their 70s and 80 s encoded semantic knowledge linked to animals and fruits at a time when society was not exposed to current modernisations [e.g., when animals mainly had a utilitarian function (Fogle, 1999) and when imported fruits were not as popular as endemic fruits]. As a consequence, we should not exclude that crosssectional differences between younger and older adults might be due to multiple concurrent factors related to neurological processing as well as sociocultural differences. However, when global and age-specific ratings for word valence (Warriner et al., 2013) were compared (this was done for words in positions 15 , where a significant group difference had emerged), no major deviation was found (Table 3), suggesting that, as far as these words are concerned, age does not seem to be associated with differences in valence attribution.

We also analysed the pattern of differences associated with SRO in a more exploratory way, following the principles of graph theory. This framework has already been used to analyse performance on the CFT, but only with nodes representing words and edges representing word-to-word, not feature-tofeature associations (Lerner et al., 2009; Goñi et al., 2011; Bertola et al., 2014). Operationalising CFT performance as a network of semantic and non-semantic features, SRO was characterised by higher degree and lower betweenness centrality at a liberal $p$-value $<0.05$. Nodal degree, a simple metric of direct centrality, was higher in older adults, albeit not exclusively limited to edges toward semantic nodes. The number of edges between SRO and semantic features did not differ between the two groups and older adults had more often an edge between SRO and both semantic (recognition time) and non-semantic (graphemes count, syllables count and dictionary orthographic Levenshtein distance) nodes. Although these three latter features are devoid of semantic information (i.e., the number of letters and syllables and the number of existing words differing by one grapheme do not convey any semantic content) they do nonetheless show important connections with SM processing. Shorter words, for instance, tend to be acquired earlier in life (Euniewska et al., 2019) and it is also known that words may activate the semantic information linked to their orthographic neighbourhood (Forster and Hector, 2002). Our findings thus suggest that semantic retrieval in older adults relies on additional lexical properties that are not semantic per se, but are of support in facilitating or expanding processing linked to SM retrieval. Conversely, although SRO betweenness centrality was positively correlated with SRO degree (Table 4B), it was lower among older adults. Although calculated in relation to each individual node, this metric captures a form of nodal centrality associated with the
TABLE 4 | Metrics calculated in association with the "serial recall order" node (A) and metric-to-metric associations.

\begin{tabular}{lccc}
\hline Variable & Younger Adults & Older Adults & $\boldsymbol{P}$ \\
\hline (A) SRO Nodal Metrics & & & \\
Degree & $4.24(2.24)$ & $5.29(3.17)$ & $0.041^{\star}$ \\
Betweenness Centrality & $0.09(0.12)$ & $0.05(0.06)$ & $0.049^{\star}$ \\
Global Efficiency & $0.54(0.15)$ & $0.56(0.19)$ & 0.353 \\
Local Efficiency & $0.67(0.32)$ & $0.67(0.36)$ & 0.981 \\
(B) Correlations Among SRO Metrics & & \\
& Degree & Local Efficiency & Global Efficiency \\
Local Efficiency & $0.369^{\star \star \star}$ & & \\
Global Efficiency & $0.870^{\star \star \star}$ & $0.397^{\star \star \star}$ & \\
Betweenness Centrality & $0.397^{\star \star \star}$ & -0.167 & $0.420^{\star \star \star}$ \\
\hline
\end{tabular}

(A) Means and standard deviations are indicated. Inferential models are described in text.

(B) Pearson's coefficients of correlation are reported.

$*: p<0.05 ;{ }^{* * *}: p<0.001$

whole graph, quantifying the proportion of times the node of interest is part of the shortest path connecting any two nodes. Lower centrality in older adults indicates that, in this group, SRO played the role of mediator node a fewer number of times. Vice versa the role of SRO within the graph of younger adults tended to control and channel the statistical link among features significantly more often.

In summary, the use of correlational measures representing the association between SRO and semantic processing showed that older adults retrieve words tagging semantic content in a way that is emotionally more neutral and of increasing lexical and semantic richness and difficulty. This was not observed homogeneously for all aspects of semantic processing, but emerged only for certain features. The two approaches to data analysis were based on distinct profiles of association: $z$-transformed correlation coefficients were analysed as continuous outcome variables, while the associative links at the basis of the graphs were binarised after the application of a cut-off. This is probably the main reason why the features distinguishing the two groups differed between the two approaches. A trend of similarity, however, was observed across methodologies (see legend in Figure 5), ruling out sharp differences between the two methods and helping define in more detail the angle from which each pattern can provide independent information.

The goal of this study was to propose a novel approach to the analysis of the CFT. While a significant correlation was found between standard CFT performance and performance on the Digit Cancellation Test (indicating a link with speed of processing), none of the significant findings showed an association with performance on tests of executive functioning (e.g., Stroop Interference test) or processing speed (e.g., Digit Cancellation test), supporting the idea that the correlational operationalisation of target variables is less influenced by supporting/intervenient factors than standard CFT scoring. The outcome emerging from the direct modelling of correlational metrics was significantly associated with performance on a test of SM that is known to be minimally influenced by processing speed 
and executive functioning (the Pyramids and Palm Trees Test). These results provide further confirmatory evidence and suggest that, of the various semantic descriptors, valence appears to be that most susceptible to the effects of ageing.

A series of potential limitations is recognised. First, the number and variety of semantic and non-semantic features was the result of an arbitrary choice based on linguistic diversity and availability of reference ratings. Second, ratings were derived from diverse populations of native English speakers and were not exclusively based on British participants. Although variability undoubtedly exists across countries and across regional areas (e.g., the concept of "animal" in rural, coastal or urban areas) in the lexicon of the two categories explored in this study, we argue that this would not result in group-level differences in trends of correlation found in association with SRO. This is, however, a methodological aspect of further improvement. Third, although we combined animal and fruit entries to maximise the number of observations at the basis of the correlation coefficients, categories normally used as part of this test may show different levels of variability in their semantic features (Stokholm et al., 2013). The significant difference found in relation to positions $1-5$ for valence was replicated for the "animals" category while a trend only emerged for the "fruits" category. We posit that this is linked to a larger variability in valence for the "animals" category (i.e., ranging from WASP: 2.71 to PANDA: 7.55 , variance $=0.94$ ) than for the "fruits" category (i.e., ranging from HAW: 4.35 to RASPBERRY: 7.30, variance $=0.18)$. "Animals" is among the most common categories used as part of the CFT, i.e., it is included in the "Addenbrooke's Cognitive Examination Revised" and in the "Consortium to Establish a Registry for Alzheimer's Disease" neuropsychological batteries. The findings of this study indicate that it is a category that offers a sufficiently sized variability to enable age differences in SM processing to emerge. Fourth, when the performance was subdivided into 5-word segments, a between-group difference was found only for the first segment. While this contributes to describing age-related trends, it is fair to note that this finding does not exploit the full lexical repertoire of the cohort, as it is based on the analysis of 900 words only ( 5 words $\times 90$ participants $\times 2$ categories), equal to only $29.4 \%$ of the total number of valid entries. Fifth, the sample was limited to 90 adults, a number that is insufficient to detect effects of small size. Sixth, although we had defined a stringent set of exclusions criteria to minimise the chances of recruiting ineligible participants, there are further neurological and psychological aspects uninvestigated in this study that may have contributed to account for part of the variability in the outcome measures. These include, for instance, genetic mechanisms (Savage et al., 2018), situational physiological variables (e.g., state anxiety/stress due to testing, mild partial sleep deprivation) and motivational factors. As far as motivation is concerned, however, although we did not administer any instrument explicitly designed to measure this process, a close inspection of individual performances on the Stroop Test (a task characterised by high cognitive demands) suggests sufficient levels of dedication put in this task by each participants. Finally, it is also worth noting that diagnoses were made based on the classification of uncorrected neuropsychological scores. Arguably, the introduction of corrected scores derived from normative data would improve diagnostic confidence and minimise the impact played by intervenient variables such as cognitive reserve.

Although this pattern of findings is preliminary at best, it warrants further attention to be paid to this theoretical framework. The additional findings obtained with the application of graph theory were significant at a more lenient threshold $(p<0.05)$ and are of exploratory relevance, given the novelty of the approach to feature-to-feature analyses. More work is needed to put additional aspects of this methodology to the test. This includes the study of test-retest reliability, its neuroimaging/neurophysiological correlates to verify construct validity, and the study of the influence additional demographic variables of neurological relevance may have, e.g., the mechanisms of cognitive reserve and plasticity. We anticipate that methods based on artificial intelligence (e.g., machine learning) could be an excellent route to process the large amount of correlational measures emerging from this procedures for a better characterisation of features that are of clinical relevance. Along the same lines, further methodological choices can be introduced to enrich the description of the link between SRO and semantic/non-semantic features, for instance the definition and assessment of Markov-Chain models to characterise in more detail the sequence of words generated during CFT. Further methodological steps could exploit the opportunity offered by statistics to isolate sources of variability by regressing out covariates of no interest or by applying latent-variable modelling to identify variables that cannot be directly measured.

This study investigated CFT performance in a group of adults with no neurological conditions. As a consequence, the extent to which this approach could be of help in clinical populations is still undetermined. Since, however, the methodology includes multiple outcome variables that are somewhat complementary to one another, these could be sensitive descriptors that could help detect very subtle neurological changes in SM or linguistic functioning (e.g., those that may occur during the pre-clinical phases of neurodegenerative conditions such as Alzheimer's disease or frontotemporal lobar degeneration). Studies carried out in clinical populations are warranted to estimate the usefulness of this method in a clinical setting, as well as to define the possible use of computational algorithms to facilitate clinical use and adoption of this more innovative scoring approach.

In conclusion, these findings suggest that the application of our scoring methodology generates correlational measures that can be useful at describing SM according to multiple thematic and graph theory-informed metrics. Proof-of-concept analyses to test this scoring approach reveal that consolidation of SM typically occurring in normal ageing is detectable and characterisable with this approach. Of the 20 metrics analysed in this study, three yielded a significant difference suggesting an effect that is not general but specific to certain properties of SM. Similarly, it is expected that the same methodology might be effective at characterising decline of SM as seen in behavioural and neurodegenerative conditions. 


\section{DATA AVAILABILITY STATEMENT}

The raw data supporting the conclusions of this article will be made available by the authors, without undue reservation.

\section{ETHICS STATEMENT}

The studies involving human participants were reviewed and approved by the Regional Ethics Committee of Yorkshire and Humber, reference number 05/Q1104/129. The participants provided their written informed consent to participate in this study.

\section{AUTHOR CONTRIBUTIONS}

MDM conceived and designed the study, contributed to the literature search, data analysis, data interpretation, writing of the report, data curation, and contributed to the tables and the figures. DJB contributed to data interpretation. AV contributed to data collection and data interpretation. All authors contributed to the article and approved the submitted version.

\section{REFERENCES}

Abbassi, E., Kahlaoui, K., Wilson, M. A., and Joanette, Y. (2011). Processing the emotions in words: the complementary contributions of the left and right hemispheres. Cogn. Affect. Behav. Neurosci. 11, 372-385. doi: 10.3758/s13415011-0034-1

Aita, S. L., Beach, J. D., Taylor, S. E., Borgogna, N. C., Harrell, M. N., and Hill, B. D. (2019). Executive, language, or both? An examination of the construct validity of verbal fluency measures. Appl. Neuropsychol. Adult 26, 441-451. doi: 10.1080/23279095.2018.1439830

Amieva, H., Le Goff, M., Millet, X., Orgogozo, J. M., Pérès, K., Barberger-Gateau, P., et al. (2008). Prodromal Alzheimer's disease: successive emergence of the clinical symptoms. Ann. Neurol. 64, 492-498. doi: 10.1002/ana.21509

Axelrod, B. N., and Wall, J. R. (2007). Expectancy of impaired neuropsychological test scores in a non-clinical sample. Int. J. Neurosci. 117, 1591-1602. doi: 10.1080/00207450600941189

Berlingeri, M., Danelli, L., Bottini, G., Sberna, M., and Paulesu, E. (2013). Reassessing the HAROLD model: is the hemispheric asymmetry reduction in older adults a special case of compensatory-related utilisation of neural circuits? Exp. Brain Res. 224, 393-410. doi: 10.1007/s00221-012-3319-x

Bertola, L., Mota, N. B., Copelli, M., Rivero, T., Satler Diniz, B., Romano-Silva, M. A., et al. (2014). Graph analysis of verbal fluency test discriminate between patients with Alzheimer's disease, mild cognitive impairment and normal elderly controls. Front. Aging Neurosci. 6:185. doi: 10.3389/fnagi.2014.00185

Bertoux, M., Duclos, H., Caillaud, M., Segobin, S., Merck, C., de La Sayette, V., et al. (2020). When affect overlaps with concept: emotion recognition in semantic variant of primary progressive aphasia. Brain 143, 3850-3864. doi: 10.1093/ brain/awaa313

Binder, L. M., Iverson, G. L., and Brooks, B. L. (2009). To err is human: "abnormal” neuropsychological scores and variability are common in healthy adults. Arch. Clin. Neuropsychol. 24, 31-46. doi: 10.1093/arclin/acn001

Biundo, R., Gardini, S., Caffarra, P., Concari, L., Martorana, D., Neri, T. M., et al. (2011). Influence of APOE status on lexical-semantic skills in mild cognitive impairment. J. Int. Neuropsychol. Soc. 17, 423-430. doi: 10.1017/ s135561771100021x

\section{FUNDING}

This research was supported by Neurocare (United Kingdom), under Grant agreement No. 181924 to MDM and AV, and by Alzheimer's Research United Kingdom, under the Pump Priming Grant scheme to MDM.

\section{ACKNOWLEDGMENTS}

We would like to thank Katija Khan, William J. McGeown, and Chiara Guerrini for administering the neuropsychological assessment of study participants and Roberta Biundo for the calculation of typicality ratings. These scores are available from the University of Hull (Kingston Upon Hull, United Kingdom) Digital Repository (https://hydra.hull.ac.uk/resources/hull:5713). We would also like to express our gratitude to Laura M. Wright for her careful revision of the manuscript.

\section{SUPPLEMENTARY MATERIAL}

The Supplementary Material for this article can be found online at: https://www.frontiersin.org/articles/10.3389/fnagi. 2021.678588/full\#supplementary-material

Brysbaert, M., Mandera, P., McCormick, S. F., and Keuleers, E. (2019). Word prevalence norms for 62,000 English lemmas. Behav. Res. Methods 51, 467-479. doi: 10.3758/s13428-018-1077-9

Brysbaert, M., Warriner, A. B., and Kuperman, V. (2014). Concreteness ratings for 40 thousand generally known English word lemmas. Behav. Res. Methods 46, 904-911. doi: 10.3758/s13428-013-0403-5

Bullmore, E., and Sporns, O. (2009). Complex brain networks: graph theoretical analysis of structural and functional systems. Nat. Rev. Neurosci. 10, 186-198. doi: $10.1038 / \mathrm{nrn} 2575$

Craik, F. I. M. (1983). On the transfer of information from temporary to permanent memory. Philos. Trans. R. Soc. Lond. B Biol. Sci. 302, 341-359. doi: 10.1098/rstb. 1983.0059

Crowe, S. F. (1998). Decrease in performance on the verbal fluency test as a function of time: evaluation in a young healthy sample. J. Clin. Exp. Neuropsychol. 20, 391-401. doi: 10.1076/jcen.20.3.391.810

Della Sala, S., Laiacona, M., Spinnler, H., and Ubezio, C. (1992). A cancellation test: its reliability in assessing attentional deficits in Alzheimer's disease. Psychol. Med. 22, 885-901. doi: 10.1017/s0033291700038460

Dubois, B., Villain, N., Frisoni, G. B., Rabinovici, G. D., Sabbagh, M., Cappa, S., et al. (2021). Clinical diagnosis of Alzheimer's disease: recommendations of the International Working Group. Lancet 20, 484-496. doi: 10.1016/s14744422(21)00066-1

Dufau, S., Grainger, J., Midgley, K. J., and Holcomb, P. J. (2015). A thousand words are worth a picture: snapshots of printed-word processing in an event-related potential megastudy. Psychol. Sci. 26, 1887-1897. doi: 10.1177/ 0956797615603934

Elgamal, S. A., Roy, E. A., and Sharratt, M. T. (2011). Age and verbal fluency: the mediating effect of speed of processing. Can. Geriatr. J. 14, 66-72. doi: 10.5770/cgj.v14i3.17

Fogle, B. (1999). The changing roles of animals in Western society: influences upon and from the veterinary profession. Anthrozoös 12, 234-239. doi: 10.2752/ 089279399787000084

Folstein, M. F., Folstein, S. E., and McHugh, P. R. (1975). "Mini-mental state". A practical method for grading the cognitive state of patients for the clinician. J. Psychiatr. Res. 12, 189-198. doi: 10.1016/0022-3956(75)90026-6 
Forbes-McKay, K. E., Ellis, A. W., Shanks, M. F., and Venneri, A. (2005). The age of acquisition of words produced in a semantic fluency task can reliably differentiate normal from pathological age related cognitive decline. Neuropsychologia 43, 1625-1632. doi: 10.1016/j.neuropsychologia.2005.01.008

Forster, K. I., and Hector, J. (2002). Cascaded versus noncascaded models of lexical and semantic processing: the turple effect. Mem. Cognit. 30, 1106-1117. doi: 10.3758/bf03194328

Gibbons, L. E., Carle, A. C., Mackin, R. S., Harvey, D., Mukherjee, S., Insel, P., et al. (2012). A composite score for executive functioning, validated in Alzheimer's Disease Neuroimaging Initiative (ADNI) participants with baseline mild cognitive impairment. Brain Imaging Behav. 6, 517-527. doi: 10.1007/ s11682-012-9176-1

Gilet, A. L., Grühn, D., Studer, J., and Labouvie-Vief, G. (2012). Valence, arousal, and imagery ratings for 835 French attributes by young, middle-aged, and older adults: the French Emotional Evaluation List (FEEL). Eur. Rev. Appl. Psychol. 62,173-181. doi: 10.1016/j.erap.2012.03.003

Goñi, J., Arrondo, G., Sepulcre, J., Martincorena, I., Vélez de Mendizábal, N., Corominas-Murtra, B., et al. (2011). The semantic organization of the animal category: evidence from semantic verbal fluency and network theory. Cogn. Process. 12, 183-196. doi: 10.1007/s10339-010-0372-x

Gonzalez-Burgos, L., Hernández-Cabrera, J. A., Westman, E., Barroso, J., and Ferreira, D. (2019). Cognitive compensatory mechanisms in normal aging: a study on verbal fluency and the contribution of other cognitive functions. Aging 11, 4090-4106. doi: 10.18632/aging.102040

Greenberg, D. L., Keane, M. M., Ryan, L., and Verfaellie, M. (2009). Impaired category fluency in medial temporal lobe amnesia: the role of episodic memory. J. Neurosci. 29, 10900-10908. doi: 10.1523/jneurosci.1202-09.2009

Gruenewald, P. J., and Lockhead, G. R. (1980). The free recall of category examples. J. Exp. Psychol. Hum. Learn. Mem. 6, 225-240. doi: 10.1037/0278-7393.6.3. 225

Grühn, D., and Smith, J. (2008). Characteristics for 200 words rated by young and older adults: age-dependent evaluations of German adjectives (AGE). Behav. Res. Methods 40, 1088-1097. doi: 10.3758/brm.40.4.1088

Guilmette, T. J., Sweet, J. J., Hebben, N., Koltai, D., Mahone, E. M., Spiegler, B. J., et al. (2020). American Academy of Clinical Neuropsychology consensus conference statement on uniform labeling of performance test scores. Clin. Neuropsychol. 34, 437-453. doi: 10.1080/13854046.2020.1722244

Hargreaves, I. S., Leonard, G. A., Pexman, P. M., Pittman, D. J., Siakaluk, P. D., and Goodyear, B. G. (2012). The neural correlates of the body-object interaction effect in semantic processing. Front. Hum. Neurosci. 6:22. doi: 10.3389/fnhum. 2012.00022

Hoaglin, D. C., Iglewicz, B., and Tukey, J. W. (1986). Performance of some resistant rules for outlier labeling. J. Am. Stat. Assoc. 81, 991-999. doi: 10.1080/01621459. 1986.10478363

Hurks, P. P. M., Hendriksen, J. G. M., Vles, J. S. H., Kalff, A. C., Feron, F. J. M., Kroes, M., et al. (2004). Verbal fluency over time as a measure of automatic and controlled processing in children with ADHD. Brain Cogn. 55, 535-544. doi: 10.1016/j.bandc.2004.03.003

Koen, J. D., and Rugg, M. D. (2019). Neural dedifferentiation in the aging brain. Trends Cogn. Sci. 23, 547-559. doi: 10.1016/j.tics.2019.04.012

Kuperman, V., Stadthagen-Gonzalez, H., and Brysbaert, M. (2012). Age-ofacquisition ratings for 30,000 English words. Behav. Res. Methods 44, 978-990. doi: 10.3758/s13428-012-0210-4

Lambon Ralph, M. A., Jefferies, E., Patterson, K., and Rogers, T. T. (2017). The neural and computational bases of semantic cognition. Nat. Rev. Neurosci. 18, 42-55. doi: $10.1038 / \mathrm{nrn} .2016 .150$

Lerner, A. J., Ogrocki, P. K., and Thomas, P. J. (2009). Network graph analysis of category fluency testing. Cog. Behav. Neurol. 22, 45-52. doi: 10.1097/wnn. ob013e318192ccaf

Łuniewska, M., Wodniecka, Z., Miller, C. A., Smolík, F., Butcher, M., Chondrogianni, V., et al. (2019). Age of acquisition of 299 words in seven languages: American English, Czech, Gaelic, Lebanese Arabic, Malay, Persian and Western Armenian. PLoS One 14:e0220611. doi: 10.1371/journal.pone. 0220611

Majerus, S., and D'Argembeau, A. (2011). Verbal short-term memory reflects the organization of long-term memory. Further evidence from short-term memory for emotional words. J. Mem. Lang. 64, 181-197. doi: 10.1016/j.jml.2010.10.003
Mandera, P., Keuleers, E., and Brysbaert, M. (2020). Recognition times for 62 thousand English words: data from the English Crowdsourcing project. Behav. Res. Methods 52, 741-760. doi: 10.3758/s13428-019-01272-8

Meyer, O. L., Eng, C. W., Ko, M. J., Chan, M. L., Ngo, U., Gilsanz, P., et al. (2020). Generation and age of immigration on later life cognitive performance in KHANDLE. Int. Psychogeriatr. doi: 10.1017/S1041610220003774 **VPQ,

Monnier, C., and Syssau, A. (2008). Semantic contribution to verbal short-term memory: are pleasant words easier to remember than neutral words in serial recall and serial recognition? Mem. Cognit. 36, 35-42. doi: 10.3758/mc.36.1.35

Murphy, D. H., and Castel, A. D. (2020). Age-related similarities and differences in the components of semantic fluency: analyzing the originality and organization of retrieval from long-term memory. Neuropsychol. Dev. Cogn. B Aging Neuropsychol. Cogn. doi: 10.1080/13825585.2020.1817844**VPQ,

Murray, W. S., and Forster, K. I. (2004). Serial mechanisms in lexical access: the rank hypothesis. Psychol. Rev. 111, 721-756. doi: 10.1037/0033-295x.111.3.721

Nyberg, L., Bäckman, L., Erngrund, K., Olofsson, U., and Nilsson, L. G. (1996). Age differences in episodic memory, semantic memory, and priming: relationships to demographic, intellectual, and biological factors. J. Gerontol. B Psychol. Sci. Soc. Sci. 51, 234-240. doi: 10.1093/geronb/51b.4.p234

Nyberg, L., Maitland, S. B., Rönnlund, M., Bäckman, L., Dixon, R. A., Wahlin, $\AA$, et al. (2003). Selective adult age differences in an age-invariant multifactor model of declarative memory. Psychol. Aging 18, 149-160. doi: 10.1037/08827974.18.1.149

Pais, R., Ruano, L., Carvalho, O. P., and Barros, H. (2020). Global cognitive impairment prevalence and incidence in community dwelling older adults-A systematic review. Geriatrics 5:84. doi: 10.3390/geriatrics5040084

Pakhomov, S. V. S., Hemmy, L. S., and Lim, K. O. (2012). Automated semantic indices related to cognitive function and rate of cognitive decline. Neuropsychologia 50, 2165-2175. doi: 10.1016/j.neuropsychologia.2012.05.016

Park, D. C., Lautenschlager, G., Hedden, T., Davidson, N. S., Smith, A. D., and Smith, P. K. (2002). Models of visuospatial and verbal memory across the adult life span. Psychol. Aging 17, 299-320. doi: 10.1037/0882-7974.17.2.299

Patterson, J. (2011). "Verbal fluency," in Encyclopedia of Clinical Neuropsychology, eds J. S. Kreutzer, J. DeLuca, and B. Caplan (New York, NY: Springer), doi: 10.1007/978-0-387-79948-3_1423

Payton, N. M., Rizzuto, D., Fratiglioni, L., Kivipelto, M., Bäckman, L., and Laukka, E. J. (2020). Combining cognitive markers to identify individuals at increased dementia risk: influence of modifying factors and time to diagnosis. J. Int. Neuropsychol. Soc. 26, 785-797. doi: 10.1017/s1355617720000272

Pexman, P. M., Muraki, E., Sidhu, D. M., Siakaluk, P. D., and Yap, M. J. (2019). Quantifying sensorimotor experience: body-object interaction ratings for more than 9,000 English words. Behav. Res. Methods 51, 453-466. doi: 10.3758/ s13428-018-1171-z

Plant, C., Webster, J., and Whitworth, A. (2011). Category norm data and relationships with lexical frequency and typicality within verb semantic categories. Behav. Res. Methods 43, 424-440. doi: 10.3758/s13428-010-0051-y

Quaranta, D., Caprara, A., Piccininni, C., Vita, M. G., Gainotti, G., and Marra, C. (2016). Standardization, clinical validation, and typicality norms of a new test assessing semantic verbal fluency. Arch. Clin. Neuropsychol. 31, 434-445. doi: 10.1093/arclin/acw034

Quaranta, D., Piccininni, C., Caprara, A., Malandrino, A., Gainotti, G., and Marra, C. (2019). Semantic relations in a categorical verbal fluency test: an exploratory investigation in mild cognitive impairment. Front. Psychol. 10:2797. doi: 10. 3389/fpsyg.2019.02797

Räling, R., Schröder, A., and Wartenburger, I. (2016). The origins of age of acquisition and typicality effects: semantic processing in aphasia and the ageing brain. Neuropsychologia 86, 80-92. doi: 10.1016/j.neuropsychologia.2016.04. 019

Reed, A. E., and Carstensen, L. L. (2012). The theory behind the age-related positivity effect. Front. Psychol. 3:339. doi: 10.3389/fpsyg.2012.00339

Rende, B., Ramsberger, G., and Miyake, A. (2002). Commonalities and differences in the working memory components underlying letter and category fluency tasks: a dual-task investigation. Neuropsychology 16, 309-321. doi: 10.1037/ 0894-4105.16.3.309

Riley, E. A., and Thompson, C. K. (2015). Training pseudoword reading in acquired dyslexia: a phonological complexity approach. Aphasiology 29, 129150. doi: $10.1080 / 02687038.2014 .955389$ 
Rönnlund, M., Nyberg, L., Bäckman, L., and Nilsson, L. G. (2005). Stability, growth, and decline in adult life span development of declarative memory: crosssectional and longitudinal data from a population-based study. Psychol. Aging 20, 3-18. doi: 10.1037/0882-7974.20.1.3

Rosch, E. (1975). Cognitive representations of semantic categories. J. Exp. Psychol. Gen. 104, 192-233. doi: 10.1037/0096-3445.104.3.192

Rubinov, M., and Sporns, O. (2010). Complex network measures of brain connectivity: uses and interpretations. Neuroimage 52, 1059-1069. doi: 10. 1016/j.neuroimage.2009.10.003

Savage, J. E., Jansen, P. R., Stringer, S., Watanabe, K., Bryois, J., de Leeuw, C. A., et al. (2018). Genome-wide association meta-analysis in 269,867 individuals identifies new genetic and functional links to intelligence. Nat. Genet. 50, 912-919. doi: 10.1038/s41588-018-0152-6

Small, B. J., Dixon, R. A., and McArdle, J. J. (2011). Tracking cognition-health changes from 55 to 95 years of age. J. Gerontol. B Psychol. Sci. Soc. Sci. 66, i153-i161. doi: 10.1093/geronb/gbq093

Söderholm, C., Häyry, E., Laine, M., and Karrasch, M. (2013). Valence and arousal ratings for 420 Finnish nouns by age and gender. PLoS One 8:e72859. doi: 10.1371/journal.pone.0072859

Sohrabi, A. (2019). Age of acquisition effect: evidence from single-word reading and neural networks. Basic Clin. Neurosci. 10, 137-146. doi: 10.32598/bcn.9.10. 120

Spaan, P. E. J. (2015). Episodic and semantic memory functioning in very old age: explanations from executive functioning and processing speed theories. Cogent. Psychol. 2:1109782. doi: 10.1080/23311908.2015.1109782

Stern, Y. (2009). Cognitive reserve. Neuropsychologia 47, 2015-2028. doi: 10.1016/ j.neuropsychologia.2009.03.004

Steyvers, M., and Tenenbaum, J. B. (2005). The large-scale structure of semantic networks: statistical analyses and a model of semantic growth. Cogn. Sci. 29, 41-78. doi: 10.1207/s15516709cog2901_3

Stokholm, J., Jørgensen, K., and Vogel, A. (2013). Performances on five verbal fluency tests in a healthy, elderly Danish sample. Neuropsychol. Dev. Cogn. B Aging Neuropsychol. Cogn. 20, 22-33. doi: 10.1080/13825585.2012.656576

Taler, V., Johns, B. T., and Jones, M. N. (2020). A large-scale semantic analysis of verbal fluency across the aging spectrum: data from the Canadian longitudinal study on aging. J. Gerontol. B Psychol. Sci. Soc. Sci. 75, e221-e230. doi: 10.1093/ geronb/gbz003

van Heuven, W. J. B., Mandera, P., Keuleers, E., and Brysbaert, M. (2014). SUBTLEX-UK: a new and improved word frequency database for British English. Q. J. Exp. Psychol. 67, 1176-1190. doi: 10.1080/17470218.2013.850521

Venneri, A., Jahn-Carta, C., De Marco, M., Quaranta, D., and Marra, C. (2018). Diagnostic and prognostic role of semantic processing in preclinical Alzheimer's disease. Biomark. Med. 12, 637-651. doi: 10.2217/bmm-2017-0324

Venneri, A., McGeown, W. J., Biundo, R., Mion, M., Nichelli, P., and Shanks, M. F. (2011). The neuroanatomical substrate of lexical-semantic decline in MCI APOE $\varepsilon 4$ carriers and noncarriers. Alzheimer Dis. Assoc. Disord. 25, 230-241. doi: 10.1097/wad.0b013e318206f88c

Venneri, A., Mitolo, M., and De Marco, M. (2016). Paradigm shift: semantic memory decline as a biomarker of preclinical Alzheimer's disease. Biomark. Med. 10, 5-8. doi: 10.2217/bmm.15.53

Venneri, A., Molinari, M. A., Pentore, R., Cotticelli, B., Nichelli, P., and Caffarra, P. (1992). Shortened Stroop color-word test: its application in Alzheimer's disease. Neurobiol. Aging 13, S3-S4. doi: 10.1016/0197-4580(92)90135-K
Verhaeghen, P. (2003). Aging and vocabulary score: a meta-analysis. Psychol. Aging 18, 332-339. doi: 10.1037/0882-7974.18.2.332

Vita, M. G., Marra, C., Spinelli, P., Caprara, A., Scaricamazza, E., Castelli, D., et al. (2014). Typicality of words produced on a semantic fluency task in amnesic mild cognitive impairment: linguistic analysis and risk of conversion to dementia. J. Alzheimers Dis. 42, 1171-1178. doi: 10.3233/jad-140570

Vonk, J. M. J., Flores, R. J., Rosado, D., Qian, C., Cabo, R., Habegger, J., et al. (2019a). Semantic network function captured by word frequency in nondemented APOE $\varepsilon 4$ carriers. Neuropsychology 33, 256-262. doi: 10.1037/ neu0000508

Vonk, J. M. J., Jonkers, R., Hubbard, H. I., Gorno-Tempini, M. L., Brickman, A. M., and Obler, L. K. (2019b). Semantic and lexical features of words dissimilarly affected by non-fluent, logopenic, and semantic primary progressive aphasia. J. Int. Neuropsychol. Soc. 25, 1011-1022. doi: 10.1017/ s1355617719000948

Wakefield, S. J., Blackburn, D. J., Harkness, K., Khan, A., Reuber, M., and Venneri, A. (2018). Distinctive neuropsychological profiles differentiate patients with functional memory disorder from patients with amnestic-mild cognitive impairment. Acta Neuropsychiatr. 30, 90-96. doi: 10.1017/neu.2017.21

Warriner, A. B., Kuperman, V., and Brysbaert, M. (2013). Norms of valence, arousal, and dominance for 13,915 English lemmas. Behav. Res. Methods 45, 1191-1207. doi: 10.3758/s13428-012-0314-x

Wongupparaj, P., Kumari, V., and Morris, R. G. (2015). A cross-temporal metaanalysis of Raven's progressive matrices: age groups and developing versus developed countries. Intelligence 49, 1-9. doi: 10.1016/j.intell.2014.11.008

Woods, D. L., Wyma, J. M., Herron, T. J., and Yund, E. W. (2016). Computerized analysis of verbal fluency: normative data and the effects of repeated testing, simulated malingering, and traumatic brain injury. PLoS One 11:e0166439. doi: 10.1371/journal.pone.0166439

Yarkoni, T., Balota, D., and Yap, M. (2008). Moving beyond Coltheart's N: a new measure of orthographic similarity. Psychon. Bull. Rev. 15, 971-979. doi: 10. 3758/pbr.15.5.971

Zar, J. H. (2005). “Spearman rank correlation: overview," in Encyclopaedia of Biostatistics, eds P. Armitage and T. Colton (Hoboken, NJ: John Wiley and Sons, Ltd), 1-9.

Conflict of Interest: The authors declare that the research was conducted in the absence of any commercial or financial relationships that could be construed as a potential conflict of interest.

Publisher's Note: All claims expressed in this article are solely those of the authors and do not necessarily represent those of their affiliated organizations, or those of the publisher, the editors and the reviewers. Any product that may be evaluated in this article, or claim that may be made by its manufacturer, is not guaranteed or endorsed by the publisher.

Copyright (c) 2021 De Marco, Blackburn and Venneri. This is an open-access article distributed under the terms of the Creative Commons Attribution License (CC BY). The use, distribution or reproduction in other forums is permitted, provided the original author(s) and the copyright owner(s) are credited and that the original publication in this journal is cited, in accordance with accepted academic practice. No use, distribution or reproduction is permitted which does not comply with these terms. 